\title{
Recovering Singularities of a Potential from Singularities of Scattering Data
}

\author{
Allan Greenleaf ${ }^{1 \star}$ and Gunther Uhlmann ${ }^{2 \star \star}$ \\ ${ }^{1}$ Department of Mathematics, University of Rochester, Rochester, New York 14627, U.S.A. \\ 2 Department of Mathematics, University of Washington, Seattle, Washington 98195, U.S.A.
}

Received October 14, 1992; in revised form February 23, 1993

\begin{abstract}
In this paper we show that the leading singularities of certain potentials can be determined from the leading singularities of the backscattering (as well as other determined sets of scattering data). The potentials in question are conormal with respect to smooth surfaces of arbitrary dimension; the restrictions on their orders allow for unbounded potentials in all dimension greater than or equal to three.
\end{abstract}

\section{Introduction}

Let $q(x)$ be a real-valued, compactly supported potential on $\mathbb{R}^{n}, n \geqq 3$, and $a(\lambda, \theta, \omega), \lambda \in \mathbb{R}, \theta, \omega \in S^{n-1}$, the scattering amplitude of $q(x)$. The nonlinear transform $q(x) \leadsto a(\lambda, \theta, \omega)$ is overdetermined and there has been much interest in the inverse problem of determining $q(x)$ from $a(\lambda, \theta, \omega)$ and the restrictions of $a$ to subsets of $\mathbb{R} \times S^{n-1} \times S^{n-1}$, e.g., [BC, ER, HN, No, N]. In this paper we will be interested in formally determined ( $n$-dimensional) sets of scattering data; moreover, we will work in the time domain, i.e., with the scattering kernel,

$$
\alpha(s, \theta, \omega)=c_{n} \int e^{i s \lambda} \lambda^{\frac{n-1}{2}} \overline{a(\lambda, \theta, \omega)} d \lambda .
$$

The class of $q$ 's considered will be those conormal to a smooth submanifold $S \subset \mathbb{R}^{n}$ of arbitrary codimension $k$. The inverse problem solved consists in determining $S$ and the symbol of $q(x)$ from the leading singularities of the scattering data. The strongest singularity of the full scattering kernel $\alpha(s, \theta, \omega)$ is of course the peak scattering; we show that for the class of potentials considered here, $\alpha(s, \theta, \omega)$ is, away from the contribution of the tangential rays, a sum of the peak scattering and a (weaker) lagrangian distribution associated with a reflected lagrangian $\hat{\Lambda}_{-} \subset T^{*}\left(\mathbb{R} \times S^{n-1} \times S^{n-1}\right)$. It is the restriction of this reflected component of

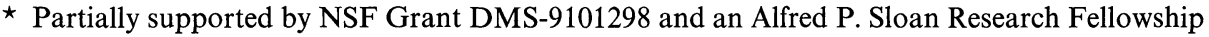
$\star \star$ Partially supported by NSF Grant DMS-9100178
} 
$\alpha(s, \theta, \omega)$ to various $n$-dimensional submanifolds of $\mathbb{R} \times S^{n-1} \times S^{n-1}$ which we show determines $S$ and the symbol of $q$ at $S$.

A particularly interesting case of our results is that of $q(x)$ having a Heavisidetype singularity across a smooth hypersurface; the location and size of the jump can then be determined from $\left.\alpha\right|_{\mathbb{B}}$, where $\mathbb{B}$ is the backscattering data,

$$
\mathbb{B}=\{(s, \theta, \omega): \theta=-\omega\} .
$$

More precisely, we prove the following; a more detailed statement, as well as the extension to other, possibly time-dependent sets of scattering data, can be found in Sect. 4.

Theorem 0.1. Let $S \subset \mathbb{R}^{n}$ be smooth of codimension $k$ and $q(x)$ conormal of order $\mu$ to $S$, with

$$
\begin{aligned}
& \mu<-\max \left(\frac{(n-2)}{n} k, k-1\right), \quad n \geqq 5 \text { and } \\
& \mu<-\max \left(\frac{k}{2}, k-1\right), \quad n=3,4 .
\end{aligned}
$$

Then $S$ and the principal symbol of $q(x)$ are determined by the singularities of the backscattering $\left.\alpha\right|_{\mathbb{B}}$.

The restriction that the order of $q(x)$ be less than $-\frac{n-2}{n} k$ or $-k / 2$, respectively, insures that the scattering kernel is defined ( $[\mathrm{P}])$; the restriction $\mu<1-k$ is needed so that the operator $\square^{-1} M_{q}$ considered in the proof is slightly smoothing.

Working in the frequency domain (i.e., with $a(\lambda, \theta, \omega)$ ), Prosser [Pr] gave a formal procedure to determine $q(x)$ from backscattering under a small norm assumption. In Eskin and Ralston [ER], the map from complex $q$ to the backscattering was shown to be generically a local homeomorphism with respect to certain norms. Note that in the theorem, although $q(x)$ belongs to a rather special class, there is no smallness assumption. Only the leading singularities of $q(x)$ are determined by $\left.\alpha\right|_{\mathbb{B}}$, but only the leading singularities of $\left.\alpha\right|_{\mathbb{B}}$ are needed to do this. (After the completion of this paper, J. Ralston brought to our attention the related paper of Päivärinta and Somersalo [PäS], which treats the question of recovering the singularities of the potential from the scattering amplitude as a function of all its variables. Their results are from the point of view of the Born approximation, rather than the time-domain approach taken here.)

The method of proof we use is to construct an approximate solution to the direct problem

$$
\left\{\begin{array}{l}
(\square+q(x)) u(x, t)=0 \quad \text { on } \mathbb{R}^{n+1} \\
u(x, t)=\delta(t-x \cdot \omega), \quad t \ll 0 .
\end{array}\right.
$$

It follows from the Lax-Phillips approach to scattering theory [LP, MU2] that $\alpha(s, \theta, \omega)$ can be expressed in terms of $u(x, t)$, cf. (3.34). It is crucial for our approach to incorporate the parameter $\omega$ as one of the independent variables. We construct an approximate solution $u \sim u_{0}+u_{1}$, with $u_{0}=\delta(t-x \cdot \omega)$. Away from the tangential rays, $u_{1}$ is a sum of the product-type lagrangian distributions; for $t \gg 0$, it is 
a sum of two lagrangian distributions, from which we find that, away from a small bad set,

$$
\alpha \in I^{\frac{1}{4}}\left(\hat{\Lambda}_{+}\right)+I^{\mu+\frac{k}{2}-\frac{5}{4}}\left(\hat{\Lambda}_{-}\right)
$$

where $\hat{\Lambda}_{+}, \hat{\Lambda}_{-} \subset T^{*}\left(\mathbb{R} \times S^{n-1} \times S^{n-1}\right)$ are the peak and reflected lagrangians, respectively. From this, the solution of the inverse problem follows easily. In Sect. 1 we recall and establish some basic results concerning classical and product-type conormal and lagrangian distributions. The action of operators such as $\square^{-1}$ on such classes is considered in Sect. 2 under various geometric assumptions. The first two terms of an approximate solution to the direct problem are constructed in Sect. 3; the analysis of the higher terms seems to be considerably more intricate and may only be possible under a strict convexity assumption on $S$; we hope to return to this point in the future. Finally, in Sect. 4 , the approximate solution to the direct problem is used to solve the inverse problem.

Much of this work was completed while the first author was on leave at the University of Washington; he would like to thank that institution for its hospitality and support.

\section{Spaces of Lagrangian Distributions}

In this section we recall the spaces of conormal distributions and distributions associated with either a single lagrangian or two cleanly intersecting lagrangian manifolds.

Let $X$ be an $n$-dimensional smooth manifold, and $\Lambda \subset T^{*} X \backslash 0$ a conic lagrangian manifold. The Hörmander space $I^{m}(\Lambda)$ of lagrangian distributions on $X$ associated with $\Lambda$ consists [Hö] of all locally finite sums of distributions of the form

$$
u(x)=\int_{\mathbb{R}^{N}} e^{i \phi(x, \theta)} a(x, \theta) d \theta
$$

where $\phi(x, \theta)$ is a nondegenerate phase function parametrizing $\Lambda$ and

$$
\begin{gathered}
a \in S^{m+\frac{n}{4}-\frac{N}{2}}\left(X \times \mathbb{R}^{N} \backslash 0\right)=\left\{a \in C^{\infty}\left(X \times\left(\mathbb{R}^{N} \backslash 0\right)\right):\right. \\
\left|\partial_{x}^{\alpha} \partial_{\theta}^{\beta} a(x, \theta)\right| \leqq C_{\alpha \beta K}\langle\theta\rangle^{m+\frac{n}{4}-\frac{N}{2}-|\alpha|}, \\
\left.\forall \alpha \in \mathbb{Z}_{+}^{N}, \beta \in \mathbb{Z}_{+}^{n}, x \in K \Subset X\right\} .
\end{gathered}
$$

(Here we use the standard notation $\langle\theta\rangle=\left(1+|\theta|^{2}\right)^{\frac{1}{2}}$.) For $u \in I^{m}(\Lambda)$, the wavefront set $W F(u) \subset \Lambda$.

Now let $S \subset X$ be a smooth submanifold of codimension $k$. Then the conormal bundle of $S$,

$$
N^{*} S=\left\{(x, \xi) \in T^{*} X \backslash 0: x \in S, \xi \perp T_{x} S\right\},
$$

is a lagrangian submanifold of $T^{*} X \backslash 0$; the space of distributions on $X$ conormal to $S$ is by definition

$$
I^{\mu}(S)=I^{\mu+\frac{1}{2}-\frac{n}{4}}\left(N^{*} S\right)
$$


If $h \in C^{\infty}\left(X, \mathbb{R}^{k}\right)$ is a defining function for $S$, with rank $(d h)=k$ at $S$, then $u(x) \in I^{\mu}(S) \Rightarrow$

$$
u(x)=\int_{\mathbb{R}^{k}} e^{i h(x) \cdot \theta} a(x, \theta) d \theta, \quad a \in S^{\mu}\left(X \times\left(\mathbb{R}^{k} \backslash 0\right)\right) .
$$

For example, if $\delta_{S}$ is a smooth density on $S$, then $\delta_{S} \in I^{0}(S)$, while a distribution on $X \backslash S$ having a Heaviside-type singularity at $S$ belongs to $I^{-k}(S)$. One easily sees that

$$
I^{\mu}(S) \subset L_{\mathrm{loc}}^{p}(X) \text { if } \mu<-k\left(1-\frac{1}{p}\right) .
$$

Now, let $\Lambda_{0}, \Lambda_{1} \subset T^{*} X \backslash 0$ be a cleanly intersecting pair of lagrangians in the sense of [MU1]. Thus, $\Sigma=\Lambda_{0} \cap \Lambda_{1}$ is smooth and

$$
T_{\lambda_{0}} \Sigma=T_{\lambda_{0}} \Lambda_{0} \cap T_{\lambda_{0}} \Lambda_{1}, \quad \forall \lambda_{0} \in \Sigma
$$

Associated to the pair $\left(\Lambda_{0}, \Lambda_{1}\right)$ is a class of lagrangian distributions, $I^{p, l}\left(\Lambda_{0}, \Lambda_{1}\right)$, indexed by $p, l \in \mathbb{R}$, which satisfy $W F(u) \subset \Lambda_{0} \cup \Lambda_{1}$ [MU1, GuU]. Microlocally, away from $\Sigma$,

$$
I^{p, l}\left(\Lambda_{0}, \Lambda_{1}\right) \subset I^{p+l}\left(\Lambda_{0} \backslash \Lambda_{1}\right) \quad \text { and } \quad I^{p, l}\left(\Lambda_{0}, \Lambda_{1}\right) \subset I^{p}\left(\Lambda_{1}\right) .
$$

If $Y_{2} \subset Y_{1} \subset X$ are smooth submanifolds with $\operatorname{codim}_{X}\left(Y_{1}\right)=d_{1}$, $\operatorname{codim}_{X}\left(Y_{2}\right)=d_{1}+d_{2}$, then $N^{*} Y_{1}$ and $N^{*} Y_{2}$ intersect cleanly in codimension $d_{2}$. The space of distributions on $X$ conormal to the pair $\left(Y_{1}, Y_{2}\right)$ of orders $\mu, \mu^{\prime}$ is

$$
\begin{aligned}
I^{\mu, \mu^{\prime}}\left(Y_{1}, Y_{2}\right) & =I^{\mu+\mu^{\prime}+\frac{d_{1}+d_{2}}{2}-\frac{n}{4},-\frac{d_{2}}{2}-\mu^{\prime}}\left(N^{*} Y_{1}, N^{*} Y_{2}\right) \\
& =I^{\mu+\frac{d_{1}}{2}-\frac{n}{4}, \mu^{\prime}+\frac{d_{2}}{2}}\left(N^{*} Y_{2}, N^{*} Y_{1}\right) .
\end{aligned}
$$

If one introduces local coordinates $\left(x_{1}, \ldots, x_{n}\right)$ on $X$ such that

$$
Y^{\prime}=\left\{x_{1}=\cdots=x_{d_{1}}=0\right\}=\left\{x^{\prime}=0\right\}
$$

and

$$
Y_{2}=\left\{x_{1}=\cdots=x_{d_{1}+d_{2}}=0\right\}=\left\{x^{\prime}=0, x^{\prime \prime}=0\right\},
$$

then $u(x) \in I^{\mu, \mu^{\prime}}\left(Y_{1}, Y_{2}\right)$ iff it can be written locally as

$$
u(x)=\int_{\mathbb{R}^{d_{1}+d_{2}}} e^{i\left(x^{\prime} \cdot \xi^{\prime}+x^{\prime \prime} \cdot \xi^{\prime \prime}\right)} a\left(x ; \xi^{\prime} ; \xi^{\prime \prime}\right) d \xi^{\prime} d \xi^{\prime \prime}
$$

with $a\left(x ; \xi^{\prime} ; \xi^{\prime \prime}\right)$ belongs to the product-type symbol class

$$
\begin{aligned}
& S^{\mu, \mu^{\prime}}\left(X \times\left(\mathbb{R}^{d_{1}} \backslash 0\right) \times \mathbb{R}^{d_{2}}\right)=\left\{a \in C^{\infty}:\left|\partial_{x}^{\gamma} \partial_{\xi^{\prime \prime}}^{\beta} \partial_{\xi^{\prime}}^{\alpha} a(x, \xi)\right|\right. \\
&\left.\leqq C_{\alpha \beta \gamma K}\left\langle\xi^{\prime}, \xi^{\prime \prime}\right\rangle^{\mu-|\alpha|}\left\langle\xi^{\prime \prime}\right\rangle^{\mu^{\prime}-|\beta|}\right\}
\end{aligned}
$$

We will need the following series of lemmas concerning multiplication of conormal distributions. 
Lemma 1.1. If $Y, Z \subset X$ are submanifolds with $Y \pitchfork Z$, then

$$
I^{\mu}(Y) \cdot I^{\mu^{\prime}}(Z) \subset I^{\mu, \mu^{\prime}}(Y, Y \cap Z)+I^{\mu^{\prime}, \mu}(Z, Y \cap Z) .
$$

If $u \in I^{\mu}(Y)$ satisfies: $\operatorname{supp}(u) \subset Y$, then

$$
u \cdot I^{\mu^{\prime}}(Z) \subset I^{\mu, \mu^{\prime}}(Y, Y \cap Z) .
$$

Proof. If $Y \cap Z=\emptyset$, there is nothing to prove, since $I^{\mu, \mu^{\prime}}(Y, Y \cap Z) \supset I^{\mu}(Y)$ and $I^{\mu^{\prime}, \mu}(Z, Y \cap Z) \supset I^{\mu^{\prime}}(Z)$. If $Y \cap Z \neq \emptyset$, let $x^{0} \in Y \cap Z$ and introduce local coordinates $\left(x^{\prime}, x^{\prime \prime}, x^{\prime \prime \prime}\right) \in \mathbb{R}^{d_{1}} \times \mathbb{R}^{d_{2}} \times \mathbb{R}^{n-d_{1}-d_{2}}$ near $x^{0}$ such that a) $x^{0}=0$, b) $Y=\left\{x^{\prime}=0\right\}$ and c) $Z=\left\{x^{\prime \prime}=0\right\}$. If $u(x) \in I^{\mu}(Y), u$ has the local oscillatory representation

$$
u(x)=\int_{\mathbb{R}^{d_{1}}} e^{i x^{\prime} \cdot \xi^{\prime}} a\left(x ; \xi^{\prime}\right) d \xi^{\prime}, \quad a \in S^{\mu}\left(X \times\left(\mathbb{R}^{d_{1}} \backslash 0\right)\right),
$$

and $v(x) \in I^{\mu \prime}(Z)$ has the representation

$$
v(x)=\int_{\mathbb{R}^{a_{2}}} e^{i x^{\prime \prime} \cdot \xi^{\prime \prime}} b\left(x ; \xi^{\prime \prime}\right) d \xi^{\prime \prime}, \quad b \in S^{\mu^{\prime}}\left(X \times\left(\mathbb{R}^{d_{2}} \backslash 0\right)\right),
$$

so that

$$
(u v)(x)=\int_{\mathbb{R}^{d_{1}+d_{2}}} e^{i\left(x^{\prime} \xi^{\prime}+x^{\prime \prime} \cdot \xi^{\prime \prime}\right)} a\left(x ; \xi^{\prime}\right) b\left(x ; \xi^{\prime \prime}\right) d \xi^{\prime} d \xi^{\prime \prime} .
$$

Introduce a cutoff function $\chi(t) \in C_{0}^{\infty}(\mathbb{R}), \chi \equiv 1$ for $|t| \leqq \frac{1}{2}, \chi \equiv 0$ for $|t| \geqq 1$. Then

$$
\chi\left(\frac{\left\langle\xi^{\prime \prime}\right\rangle}{\left\langle\xi^{\prime}\right\rangle}\right) a\left(x ; \xi^{\prime}\right) b\left(x ; \xi^{\prime \prime}\right) \in S^{\mu, \mu^{\prime}}\left(X \times\left(\mathbb{R}^{d_{1}} \backslash 0\right) \times \mathbb{R}^{d_{2}}\right),
$$

while

$$
(1-\chi)\left(\frac{\left\langle\xi^{\prime \prime}\right\rangle}{\left\langle\xi^{\prime}\right\rangle}\right) a\left(x ; \xi^{\prime}\right) b\left(x ; \xi^{\prime \prime}\right) \in S^{\mu^{\prime}, \mu}\left(X \times\left(\mathbb{R}^{d_{2}} \backslash 0\right) \times \mathbb{R}^{d_{1}}\right) ;
$$

making the corresponding decomposition of $u \cdot v$ yield (1.7).

If $\operatorname{supp}(u) \subset Y$, then by [Hö], $W F(u v) \subset N^{*} Y \cup N^{*}(Y \cap Z)$, so that in the above decomposition the second term belongs to $I^{\mu+\mu^{\prime}}(Y \cap Z) \subset I^{\mu, \mu^{\prime}}(Y, Y \cap Z)$, yielding (1.8). Q.E.D.

We also need the multiplicative properties of conormal distributions associated with a nested pair of submanifolds. Related results for a single submanifold are in [Pi].

Lemma 1.2. If $Y_{1} \supset Y_{2}$ are submanifolds of $X$ of codimensions $d_{1}, d_{1}+d_{2}$, respectively, $u_{1} \in I^{M_{1}}\left(Y_{1}\right)$, and $u_{2} \in I^{M_{2}}\left(Y_{2}\right)$ is microlocally supported away from $N^{*} Y_{1}$, then

$$
\begin{aligned}
u_{1} u_{2} \in I^{M^{\prime}, M^{\prime \prime}}\left(Y_{1}, Y_{2}\right), & M^{\prime}=\left(m_{1}+d_{1}\right)_{+}-d_{1}+\varepsilon \delta_{m_{1},-d_{1}}, \\
M^{\prime \prime} & =m_{2}+d_{1}, \text { any } \varepsilon>0 .
\end{aligned}
$$


Proof. Introducing on $X$ local coordinates $x=\left(x^{\prime}, x^{\prime \prime}, x^{\prime \prime \prime}\right)$ as discussed above (1.5), we have oscillatory representations

$$
u_{1}(x)=\int_{\mathbb{R}^{d_{1}}} e^{x^{\prime} \cdot \xi^{\prime}} a\left(x ; \xi^{\prime}\right) d \xi^{\prime}, \quad a \in S^{M_{1}}\left(X \times\left(\mathbb{R}^{d_{1}} \backslash 0\right)\right)
$$

and

$$
u_{2}(x)=\int_{\mathbb{R}^{d_{1}+d_{2}}} e^{i\left(x^{\prime} \cdot \xi^{\prime}+x^{\prime \prime} \cdot \xi^{\prime \prime}\right)} b\left(x ; \xi^{\prime}, \xi^{\prime \prime}\right) d \xi^{\prime} d \xi^{\prime \prime}, \quad b \in S^{m_{2}}\left(X \times\left(\mathbb{R}^{d_{1}+d_{2}} \backslash 0\right)\right)
$$

with $\operatorname{supp}(b) \subset\left\{\left|\xi^{\prime}\right| \leqq c\left|\xi^{\prime \prime}\right|\right\}$. Thus,

$$
\left(u_{1} u_{2}\right)(x)=\int_{\mathbb{R}^{d_{1}+d_{2}}}\left(a *^{\prime} b\right)\left(x ; \xi^{\prime}, \xi^{\prime \prime}\right) d \xi^{\prime} d \xi^{\prime \prime},
$$

where $a *^{\prime} b$ is the partial convolution

$$
a *^{\prime} b\left(x ; \xi^{\prime}, \xi^{\prime \prime}\right)=\int_{\mathbb{R}^{d_{1}}} a\left(x ; \eta^{\prime}\right) b\left(x ; \xi^{\prime}-\eta^{\prime}, \xi^{\prime \prime}\right) d \eta^{\prime}
$$

where $a *^{\prime} b$ is the partial convolution

$$
a *^{\prime} b\left(x ; \xi^{\prime}, \xi^{\prime \prime}\right)=\int_{\mathbb{R}^{d_{1}}} a\left(x ; \eta^{\prime}\right) b\left(x ; \xi^{\prime}-\eta^{\prime}, \xi^{\prime \prime}\right) d \eta^{\prime} .
$$

To estimate the size of $a *^{\prime} b\left(x ; \xi^{\prime}, \xi\right)$, where $\left|\xi^{\prime}\right| \geqq c\left|\xi^{\prime \prime}\right|$, note that

$$
\left|a *^{\prime} b\left(x ; \xi^{\prime}, \xi^{\prime \prime}\right)\right| \leqq c\left\langle\xi^{\prime}\right\rangle^{m_{1}} \int_{\left|\xi^{\prime}-\eta^{\prime}\right| \leqq c\left|\xi^{\prime \prime}\right|}\left\langle\xi^{\prime \prime}\right\rangle^{m_{2}} d \eta^{\prime} \leqq c\left\langle\xi, \xi^{\prime}\right\rangle^{m_{1}}\left\langle\xi^{\prime \prime}\right\rangle^{m_{2}+d_{1}}
$$

On the other hand, if $\left|\xi^{\prime}\right| \leqq c\left|\xi^{\prime \prime}\right|$,

$$
\left|a *^{\prime} b\left(x ; \xi^{\prime}, \xi^{\prime \prime}\right)\right| \leqq c\left\langle\xi^{\prime \prime}\right\rangle^{m_{2}} \int_{\left|\eta^{\prime}\right| \leqq c\left|\xi^{\prime \prime}\right|}\left\langle\eta^{\prime}\right\rangle^{m_{1}} d \eta^{\prime} \leqq c\left\langle\xi^{\prime \prime}\right\rangle^{\left(m_{1}+d_{1}\right)_{+}}+m_{2}+\varepsilon \delta_{m_{1},-d_{1}}
$$

for any $\varepsilon>0$. Thus, $a *^{\prime} b$ satisfies the correct size estimate to belong to $S^{M^{\prime}, M^{\prime \prime}}\left(X \times\left(\mathbb{R}^{d_{1}} \backslash 0\right) \times \mathbb{R}^{d_{2}}\right)$, with $M^{\prime}, M^{\prime \prime}$ as in (1.9). A derivative in $\xi^{\prime}, \partial_{\xi^{\prime}}^{\alpha}\left(a *^{\prime} b\right)$, can be represented as either $\left(\partial_{\xi^{\prime}}^{\alpha} a\right) *^{\prime} b$ or $\left.a *^{\prime} \partial_{\xi^{\prime}}^{\alpha} b\right)$. In the region $\left.\left|\xi^{\prime}\right| \geqq c\left|\xi^{\prime \prime}\right|\right\}$, we use $\partial_{\xi^{\prime}}^{\alpha} a \in S^{m_{1}-|\alpha|}$ and (1.14) to get a gain of $\left\langle\xi^{\prime}\right\rangle^{-|\alpha|}$ as long as $|\alpha| \leqq m_{1}+d_{1}$; for $|\alpha|>m_{1}+d_{1}$, we integrate by parts $|\alpha|-m_{1}-d_{1}$ times and then apply (1.14) to obtain the desired gain. On $\left\{\left|\xi^{\prime}\right| \leqq c\left|\xi^{\prime \prime}\right|\right\}$, we use $\partial_{\xi^{\prime}}^{\alpha} b \in S^{m_{2}-|\alpha|}$ and (1.15) to obtain the gain of $\left\langle\xi^{\prime \prime}\right\rangle^{-|\alpha|}=\left\langle\xi^{\prime}, \xi^{\prime \prime}\right\rangle^{-|\alpha|}$. A derivative $\partial_{\xi^{\prime \prime}}^{\beta^{\prime \prime}}$, however, can only be distributed to $b\left(x ; \xi^{\prime}, \xi^{\prime \prime}\right)$, lowering $m_{2}$ to $m_{2}-|\beta|$ and consequently yielding a gain of only $\left\langle\xi^{\prime \prime}\right\rangle^{-|\beta|}$. Thus, $a *^{\prime} b \in S^{M^{\prime}, M^{\prime \prime}}\left(X \times\left(\mathbb{R}^{d_{1}} \backslash 0\right) \times \mathbb{R}^{d_{2}}\right)$ and $u_{1} u_{2} \in I^{M^{\prime}, M^{\prime \prime}}\left(Y_{1}, Y_{2}\right)$ by (1.5). Q.E.D.

If $u_{2}$ is microlocally supported near $N^{*} Y_{1}$, we have a similar result.

Lemma 1.3. If $Y_{1}, Y_{2}$ and $u_{1}(x)$ are as above, and $u_{2} \in I^{m_{2}}\left(Y_{2}\right)$ has amplitude $b\left(x ; \xi^{\prime}, \xi^{\prime \prime}\right)$ supported in $\left\{\left|\xi^{\prime \prime}\right| \leqq c\left|\xi^{\prime}\right|\right\}$, then

$$
u_{1} u_{2} \in I^{m_{1}, m_{2}+d_{1}}\left(Y_{1}, Y_{2}\right), m_{2}<-d_{1}, m_{1}+m_{2}<-d_{1} .
$$


Proof. We repeat the calculations of the previous proof, except that in (1.13), the integral is over $\left\{\left|\xi^{\prime}-\eta^{\prime}\right| \geqq c\left|\xi^{\prime \prime}\right|\right\}$. Thus, on $\left\{\left|\xi^{\prime}\right| \geqq c\left|\xi^{\prime \prime}\right|\right\}$,

$$
\left|a *^{\prime} b\left(x ; \xi^{\prime}, \xi^{\prime \prime}\right)\right| \leqq c\left\langle\xi^{\prime}\right\rangle^{m_{1}} \int_{\left|\eta^{\prime}\right| \geqq c\left|\xi^{\prime \prime}\right|}\left\langle\eta^{\prime}, \xi^{\prime \prime}\right\rangle^{m_{2}} d \eta^{\prime} \leqq\left\langle\xi^{\prime}\right\rangle^{m_{1}}\left\langle\xi^{\prime \prime}\right\rangle^{m_{2}+d_{1}}
$$

if $m_{2}+d_{1}<0$, while on $\left\{\left|\xi^{\prime}\right| \leqq\left|\xi^{\prime \prime}\right|\right\}$,

$$
\left|a *^{\prime} b\left(x ; \xi^{\prime}, \xi^{\prime \prime}\right)\right| \leqq \int_{\left|\eta^{\prime}\right| \geqq c\left|\xi^{\prime \prime}\right|}\left\langle\eta^{\prime}\right\rangle^{m_{1}+m_{2}} d \eta^{\prime} \tau c\left\langle\xi^{\prime \prime}\right\rangle^{m_{1}+m_{2}+d_{1}}, m_{1}+m_{2}+d_{1}<0 .
$$

Q.E.D.

We also will need the action of $I^{m_{1}}\left(Y_{1}\right)$ on spaces of product-type conormal distributions.

Lemma 1.4. If $Y_{1}, Y_{2}$ are as above, $u_{1} \in I^{m_{1}}\left(Y_{1}\right)$, and $u_{2} \in I^{M^{\prime}, M^{\prime \prime}}\left(Y_{1}, Y_{2}\right)$ is supported microlocally near $N^{*} Y_{1}$, with $M^{\prime}<-d_{1}, M^{\prime} \leqq m_{1}$, then $u_{1} u_{2} \in I^{\tilde{M}^{\prime}, M^{\prime \prime}}\left(Y_{1}, Y_{2}\right), \tilde{M}^{\prime}=\max \left(\left(m_{1}+d_{1}\right)_{+}+M^{\prime}+\varepsilon \delta_{m_{1},-d_{1}}, m_{1}\right)$, and $\varepsilon>0$.

Proof. We again have the oscillatory representation (1.12) of $u_{1} u_{2}$ with $a *^{\prime} b$ given by (1.13) for $a \in S^{m_{1}}\left(X \times\left(\mathbb{R}^{d_{1}} \backslash 0\right)\right)$ but now $b \in S^{M^{\prime}, M^{\prime \prime}}\left(X \times\left(\mathbb{R}^{d_{1}} \backslash 0\right) \times \mathbb{R}^{d_{2}}\right)$; thus, $\left|a\left(x ; \xi^{\prime}\right)\right| \leqq c\left\langle\xi^{\prime}\right\rangle^{m_{1}}$ and $\left|b\left(x ; \xi^{\prime}, \xi^{\prime \prime}\right)\right| \leqq c\left\langle\xi^{\prime}\right\rangle^{M^{\prime}}\left\langle\xi^{\prime \prime}\right\rangle^{M^{\prime \prime}}$. For $\left|\xi^{\prime}\right| \leqq c\left|\xi^{\prime \prime}\right|$,

$$
\begin{aligned}
\left|a *^{\prime} b\left(x ; \xi^{\prime}, \xi^{\prime \prime}\right)\right| & \leqq c \int_{\left|\xi^{\prime}-\eta^{\prime}\right| \geqq c\left|\xi^{\prime \prime}\right|}\left\langle\eta^{\prime}\right\rangle^{m_{1}}\left\langle\xi^{\prime}-\eta^{\prime}\right\rangle^{M^{\prime}}\left\langle\xi^{\prime \prime}\right\rangle^{M^{\prime \prime}} d \eta^{\prime} \\
& \leqq c\left\langle\xi^{\prime \prime}\right\rangle^{M^{\prime \prime}} \int_{\left|\eta^{\prime}\right| \geqq c \xi^{\prime \prime} \mid}\left\langle\eta^{\prime}\right\rangle^{m_{1}+M^{\prime}} d \eta^{\prime} \\
& \leqq c\left\langle\xi^{\prime \prime}\right\rangle^{m_{1}+M^{\prime}+M^{\prime \prime}+d_{1}} \quad \text { if } m_{1}+M^{\prime}<-d_{1}
\end{aligned}
$$

If $\left|\xi^{\prime}\right| \geqq c\left|\xi^{\prime \prime}\right|$, we use the first inequality in (1.18) and then decompose the integral into three pieces, corresponding to the regions $\mathrm{I}=\left\{\left\langle\eta^{\prime}\right\rangle \leqq c\left\langle\xi^{\prime}\right\rangle\right\}$, II $=\left\{c\left\langle\xi^{\prime \prime}\right\rangle \leqq\left\langle\eta^{\prime}\right\rangle \leqq c\left\langle\xi^{\prime}\right\rangle\right\}$ and III $=\left\{\left\langle\xi^{\prime}-\eta^{\prime}\right\rangle \geqq c\left\langle\xi^{\prime \prime}\right\rangle\right\}$ we have

$$
\int_{\mathrm{I}} \leqq c\left\langle\xi^{\prime}\right\rangle^{M^{\prime}}\left\langle\xi^{\prime \prime}\right\rangle^{M^{\prime \prime}} \int_{\mathrm{I}}\left\langle\eta^{\prime}\right\rangle^{m_{1}} d \eta^{\prime} \leqq c\left\langle\xi^{\prime}\right\rangle^{\left(m_{1}+d_{1}\right)+M^{\prime}+\varepsilon \delta}\left\langle\xi^{\prime \prime}\right\rangle^{m},
$$

where $\delta=\delta_{m_{1},-d_{1}}$ and $\varepsilon>0$ is arbitrary,

$$
\begin{aligned}
\int_{\text {II }} \leqq & c\left\langle\xi^{\prime \prime}\right\rangle^{M^{\prime \prime}}\left(\left\langle\xi^{\prime}\right\rangle^{M^{\prime}} \int_{\text {II }}\left\langle\eta^{\prime}\right\rangle^{m_{1}} d \eta^{\prime}+\left\langle\xi^{\prime}\right\rangle^{m_{1}} \int_{\text {II }}\left\langle\eta^{\prime}\right\rangle^{M^{\prime}} d \eta^{\prime}\right) \\
\leqq & c\left\langle\xi^{\prime \prime}\right\rangle^{M^{\prime \prime}}\left(\left\langle\xi^{\prime}\right\rangle^{M^{\prime}} \max \left(\left\langle\xi^{\prime \prime}\right\rangle^{m_{1}+d_{1}+\varepsilon_{1}},\left\langle\xi^{\prime}\right\rangle^{m_{1}+d_{1}+\varepsilon \delta}\right)\right. \\
& \left.+\left\langle\xi^{\prime}\right\rangle^{m_{1}} \max \left(\left\langle\xi^{\prime \prime}\right\rangle^{M^{\prime \prime}+d_{1}},\left\langle\xi^{\prime}\right\rangle^{M^{\prime}+d_{1}}\right)\right) \\
\leqq & \begin{cases}c\left\langle\xi^{\prime}\right\rangle^{m_{1}}\left\langle\xi^{\prime \prime}\right\rangle^{M^{\prime}+M^{\prime \prime}+d_{1}}, & m_{1}+d_{1} \geqq 0 \\
c \max \left(\left\langle\xi^{\prime}\right\rangle^{M^{\prime}}\left\langle\xi^{\prime \prime}\right\rangle^{m_{1}+M^{\prime \prime}+d_{1}},\left\langle\xi^{\prime}\right\rangle^{m_{1}}\left\langle\xi^{\prime \prime}\right\rangle^{M^{\prime} \mid M^{\prime \prime}+d_{1}}\right), & m_{1}+d_{1}<0\end{cases} \\
\leqq & c\left\langle\xi^{\prime}\right\rangle\left\langle\xi^{\prime \prime}\right\rangle^{M^{\prime}+M^{\prime \prime}+d_{1}} \quad \text { if } m_{1} \geqq M^{\prime},
\end{aligned}
$$


and

$$
\int_{\text {III }} \leqq c \int_{\text {III }}\left\langle\eta^{\prime}\right\rangle^{m_{1}+M^{\prime}}\left\langle\xi^{\prime \prime}\right\rangle^{M^{\prime \prime}} d \eta^{\prime} \leqq c\left\langle\xi^{\prime}\right\rangle^{m_{1}+M^{\prime}+d_{1}}\left\langle\xi^{\prime \prime}\right\rangle^{M^{\prime \prime}}
$$

Since $\left(m_{1}+d_{1}\right)+\varepsilon \delta \geqq m_{1}+d_{1}$, the third term is dominated by the first; on the other hand, since $M^{\prime}+d_{1}<0$, the second term is dominated by $c\left\langle\xi^{\prime}\right\rangle^{m_{1}}\left\langle\xi^{\prime \prime}\right\rangle^{M^{\prime \prime}}$. Thus, on $\left\{\left|\xi^{\prime}\right| \geqq c\left|\xi^{\prime \prime}\right|\right\}$,

$$
\left|a *^{\prime} b\left(x ; \xi^{\prime}, \xi^{\prime \prime}\right)\right| \leqq c\left\langle\xi^{\prime}\right\rangle^{\tilde{M}^{\prime}}\left\langle\xi^{\prime \prime}\right\rangle^{M^{\prime \prime}} .
$$

The estimates (1.18) and (1.22) together imply the size estimate satisfied by an amplitude belonging to $S^{\tilde{M}^{\prime}, M^{\prime \prime}}$. As before, $\partial_{\xi^{\prime}}^{\alpha}$ can be distributed to either $a$ or $b$, while $\partial_{\xi^{\prime \prime}}^{\beta}$ must be applied to $b$, yielding the required estimate

$$
\left|\partial_{x}^{\gamma} \partial_{\xi^{\prime \prime}}^{\beta} \partial_{\xi^{\prime}}^{\alpha}\left(a *^{\prime} b\right)\right| \leqq c\left\langle\xi^{\prime}, \xi^{\prime \prime}\right\rangle^{\tilde{M}^{\prime}-|\alpha|}\left\langle\xi^{\prime \prime}\right\rangle^{M^{\prime \prime}-|\beta|},
$$

so that $u_{1} u_{2} \in I^{\tilde{M}^{\prime}, M^{\prime \prime}}\left(Y_{1}, Y_{2}\right)$. Q.E.D.

Finally, we have

Lemma 1.5. Let $Y_{1} \supset Y_{2}$ be as above, and $Y_{+} \subset X$ such that $Y_{1} \pitchfork Y_{+}$with $Y_{1} \cap Y_{+}=Y_{2}$. Let $u_{1} \in I^{m_{1}}\left(Y_{1}\right)$, and $u_{2} \in I^{M^{\prime}, M^{\prime \prime}}\left(Y_{+}, Y_{2}\right)$ be supported microlocally near $N^{*} Y_{+}$. Then,

$$
u_{1} u_{2} \in I^{M^{\prime}, \tilde{M}^{\prime \prime}}\left(Y_{+}, Y_{2}\right)+I^{\tilde{M}^{\prime}, 0}\left(Y_{+}, Y_{2}\right)+I^{m_{1}, m_{2}}\left(Y_{1}, Y_{2}\right),
$$

where $\tilde{M}^{\prime \prime}=M^{\prime \prime}+\left(m_{1}+d_{1}\right)_{+}+\varepsilon \delta_{m_{1},-d_{1}}, \tilde{M}^{\prime}=M^{\prime}+\left(m_{1}+M^{\prime \prime}+d_{1}\right)_{+}+\varepsilon \delta_{m_{1}+M^{\prime \prime},-d_{1}}$, and $m_{2}=M^{\prime}+\left(M^{\prime \prime}+d_{1}\right)_{+}+\varepsilon \delta_{M^{\prime \prime},-d_{1}}$, any $\varepsilon>0$.

Proof. We may choose the local coordinates $x=\left(x^{\prime}, x^{\prime \prime}, x^{\prime \prime \prime}\right)$ so that $Y_{1}=\left\{x^{\prime}=0\right\}$, $Y_{+}=\left\{x^{\prime \prime}=0\right\}$, and $Y_{2}=\left\{x^{\prime}=0, x^{\prime \prime}=0\right\} . u_{1}(x)$ is given by (1.10) while

$$
u_{2}(x)=\int_{\mathbb{R}^{d_{1}+d_{2}}} e^{i\left(x^{\prime} \cdot \xi^{\prime}+x^{\prime \prime} \cdot \xi^{\prime \prime}\right)} b\left(x ; \xi^{\prime \prime}, \xi^{\prime}\right) d \xi^{\prime} d \xi^{\prime \prime}, \quad b \in S^{M^{\prime}, M^{\prime \prime}}\left(X \times\left(\mathbb{R}^{d_{2}} \backslash 0\right) \times \mathbb{R}\right)
$$

with $\operatorname{supp}(b) \subset\left\{\left|\xi^{\prime \prime}\right| \leqq c\left|\xi^{\prime \prime}\right|\right\}$. (Note that $\xi^{\prime \prime}$ plays the role of the "elliptic" variable.) The product $\left(u_{1}, u_{2}\right)(x)$ is again represented by $(1.12)$, with

$$
a *^{\prime} b\left(x ; \xi_{1}, \xi_{2}\right)=\int_{\mathbb{R}^{d_{1}}} a\left(x ; \xi^{\prime}-\eta^{\prime}\right) b\left(x ; \xi^{\prime \prime}, \eta^{\prime}\right) d \eta^{\prime}
$$

It suffices to show that

$$
a *^{\prime} b \in S^{M^{\prime}, \tilde{M}^{\prime \prime}}\left(X \times\left(\mathbb{R}^{d_{2}} \backslash 0\right) \times \mathbb{R}^{d_{1}}\right)+S^{\tilde{M}^{\prime}, 0}\left(X \times\left(\mathbb{R}^{d_{2}} \backslash 0\right) \times \mathbb{R}^{d_{1}}\right)\left\{\left|\xi^{\prime}\right| \leqq c\left|\xi^{\prime \prime}\right|\right\}
$$

while

$$
a *^{\prime} b \in S^{m_{1}, m_{2}}\left(X \times\left(\mathbb{R}^{d_{1}} \backslash 0\right) \times \mathbb{R}^{d_{2}}\right) \quad \text { on }\left\{\left|\xi^{\prime \prime}\right| \leqq c\left|\xi^{\prime}\right|\right\}
$$


Where $\left|\xi^{\prime \prime}\right| \leqq c\left|\xi^{\prime}\right|$, one has

$$
\begin{aligned}
\left|a *^{\prime} b\left(x ; \xi^{\prime}, \xi^{\prime \prime}\right)\right| & \leqq c \int_{\left|\eta^{\prime}\right| \leqq c\left|\xi^{\prime \prime}\right|}\left\langle\xi^{\prime}-\eta^{\prime}\right\rangle^{m_{1}}\left\langle\eta^{\prime}, \xi^{\prime \prime}\right\rangle^{M^{\prime}}\left\langle\eta^{\prime}\right\rangle^{M^{\prime \prime}} d \eta^{\prime} \\
& \leqq c\left\langle\xi^{\prime}\right\rangle^{m_{1}}\left\langle\xi^{\prime \prime}\right\rangle^{M^{\prime}} \int_{\left|\eta^{\prime}\right| \leqq c\left|\xi^{\prime \prime}\right|}\left\langle\eta^{\prime}\right\rangle^{M^{\prime \prime}} d \eta^{\prime} \\
& \leqq c\left\langle\xi^{\prime}\right\rangle^{m_{1}}\left\langle\xi^{\prime \prime}\right\rangle^{M^{\prime}+\left(M^{\prime \prime}+d_{1}\right)_{+}+\varepsilon \delta_{M^{\prime \prime},-d_{1}}}, \text { any } \varepsilon>0,
\end{aligned}
$$

which is the correct size estimate for (1.26). Applying $\partial_{\xi^{\prime}}^{\alpha}$ to the the first factor in $a *^{\prime} b$, we lower $m_{1}$ by $|\alpha|$ and obtain a gain of $\left\langle\xi^{\prime}\right\rangle^{-|\alpha|} \simeq\left\langle\xi^{\prime}, \xi^{\prime \prime}\right\rangle^{-|\alpha|}$, while $\partial_{\xi^{\prime \prime}}^{\beta}$ must be applied to the second factor, resulting in a gain of only $\left\langle\xi^{\prime \prime}\right\rangle^{-|\beta|}$. Thus, (1.26) holds. As for (1.25), on $\left\{\left|\xi^{\prime \prime}\right| \geqq c\left|\xi^{\prime}\right|\right\}$, we have

$$
\begin{aligned}
\left|a *^{\prime} b\left(x ; \xi^{\prime}, \xi^{\prime \prime}\right)\right|= & \left|\int_{\left|\eta^{\prime}\right| \leqq c \xi^{\prime \prime} \mid} a\left(x ; \xi^{\prime}-\eta^{\prime}\right) b\left(x ; \xi^{\prime \prime}, \eta^{\prime}\right) d \eta^{\prime}\right| \\
\leqq & c \int_{\left|\eta^{\prime}\right| \leqq c\left|\xi^{\prime \prime}\right|}\left\langle\eta^{\prime}\right\rangle^{m_{1}+M^{\prime \prime}}\left\langle\xi^{\prime \prime}\right\rangle^{M^{\prime}} d \eta^{\prime} \\
& +c \int_{\left|\xi^{\prime}-\eta^{\prime}\right| \leqq \frac{1}{2}\left|\xi^{\prime}\right|}\left\langle\xi^{\prime}-\eta^{\prime}\right\rangle^{m_{1}}\left\langle\xi^{\prime \prime}\right\rangle^{M^{\prime}}\left\langle\xi^{\prime}\right\rangle^{M^{\prime \prime}} d \eta^{\prime} \\
\leqq & c\left\langle\xi^{\prime \prime}\right\rangle M^{\prime}+\left(m_{1}+M^{\prime \prime}+d_{1}\right)_{+}+\varepsilon \delta_{m_{1}+M^{\prime \prime},-d_{i}} \\
& +c\left\langle\xi^{\prime \prime}\right\rangle M^{M^{\prime}}\left\langle\xi^{\prime}\right\rangle^{M^{\prime \prime}+\left(m_{1}+d_{1}\right)_{+}+\varepsilon \delta_{m_{1},-d_{1}}},
\end{aligned}
$$

which is the correct size estimate for (1.25). The desired gain of $\left\langle\xi^{\prime \prime}\right\rangle^{-|\beta|}$ from the application of $\partial_{\xi^{\prime \prime}}^{\beta}$ follows, since $M^{\prime}$ is lowered by $|\beta|$. As in the proof of Lemma 1.2, the gain of $\left\langle\xi^{\prime}\right\rangle^{-|\alpha|}$ from $\partial_{\xi^{\prime}}^{\alpha}$ follows by lowering $M^{\prime \prime}$ to $M^{\prime \prime}-|\alpha|$ if $m_{1}+M^{\prime \prime} \geqq-d_{1}$; otherwise one integrates by parts first. Thus, (1.25) holds and the lemma is proved. Q.E.D.

\section{Action of Parametrices on Distribution Spaces}

We now consider the mapping properties of a parametrix for a pseudodifferential operator of real principal type, acting on the spaces of distributions associated with one and two lagrangians described in Sect. 1. The intended application in Sect. 3 is to the d'Alembertian on $\mathbb{R}^{n+1} \times S^{n-1}$, but the natural coordinates there do not seem convenient for establishing these results, leading us to formulate and prove them in the generality described below.

Let $P(x, D)$ be an $m^{\text {th }}$ order classical $\psi D O$, with real homogeneous principal symbol $p_{m}(x, \xi)$. Recall that $P$ is of real principal type if a) $d p_{m} \neq 0$ at $\operatorname{char}(P)=\left\{(x, \xi) \in T^{*} X \backslash 0: p_{m}(x, \xi)=0\right\}$ so that $\operatorname{char}(P)$ is smooth, and b) $\operatorname{char}(P)$ has no characteristics trapped over a compact set of $X$. Then $P(x, D)$ is locally solvable and parametrices for $P(x, D)$ were constructed in [DH, MU1]. For $(x, \xi) \in \operatorname{char}(P)$, let $\Xi_{(x, \xi)}$ be the bicharacteristic of $P(x, D)$ (i.e., integral curve of $\left.H_{p_{m}}\right)$ through $(x, \xi)$. Then the flowout canonical relation generated by $\operatorname{char}(P)$,

$$
\Lambda_{P}=\left\{(x, \xi ; y, \eta):(x, \xi) \in \operatorname{char}(P),(y, \eta) \in \Xi_{(x, \xi)}\right\},
$$


intersects the diagonal $\Delta_{T}{ }^{*}$ cleanly in codimension 1. In [MU1], it was shown that $P(x, D)$ has a parametrix $Q \in I^{\frac{1}{2}-m,-\frac{1}{2}}\left(\Delta_{T^{*} X}, \Lambda_{P}\right)$.

Proposition 2.1. Suppose $\Lambda_{0} \subset T^{*} X \backslash 0$ is a conic lagrangian intersecting char $(P)$ transversally and such that each bicharacteristics of $P$ intersects $\Lambda_{0}$ a finite number of times. Then, if $T \in I^{p, l}\left(\Delta_{T^{*} X}, \Lambda_{P}\right)$,

$$
T: I^{r}\left(\Lambda_{0}\right) \rightarrow I^{r+p, l}\left(\Lambda_{0}, \Lambda_{1}\right),
$$

where $\Lambda_{1}=\Lambda_{P} \circ \Lambda_{0}$ is the flowout from $\Lambda$ on $\operatorname{char}(P)$. Furthermore, for $(x, \xi) \in$ $\Lambda_{1} \backslash \Lambda_{0}$,

$$
\sigma(T u)(x, \xi)=\sum_{j} \sigma(T)\left(x, \xi ; y_{j}, \eta_{j}\right) \sigma(u)\left(y_{j}, \eta_{j}\right)
$$

where $\left\{\left(y_{j}, \eta_{j}\right)\right\}=\Lambda_{0} \cap \Xi_{(x, \xi)}$.

Proof. Microlocalizing and conjugating by an elliptic Fourier integral operator associated with a canonical transformation, we can assume [MU1] that $X=\mathbb{R}^{n}$ with coordinates $x=\left(x_{1}, x^{\prime}\right), \Lambda_{0}{ }^{*}=T_{0}^{*} \mathbb{R}^{n} \backslash 0, \operatorname{char}(P)=\left\{(x, \xi): \xi_{1}=0\right\}$ and thus

$$
\Lambda_{1}=\left\{\left(x_{1}, 0 ; 0, \xi^{\prime}\right): x_{1} \in \mathbb{R}, \xi^{\prime} \in \mathbb{R}^{n-1} \backslash 0\right\} .
$$

A distribution $u \in I^{r}\left(\Lambda_{0}\right)$ has the representation

$$
u(x)=\int_{\mathbb{R}^{n}} e^{i x \cdot \xi} a(x ; \xi) d \xi, \quad a \in S^{r-\frac{n}{4}}\left(\mathbb{R}^{n} \times\left(\mathbb{R}^{n} \backslash 0\right)\right),
$$

and $T \in I^{p, l}\left(\Delta_{T * \mathbb{R}^{n}}, \Lambda_{p}\right)$ has the form

$$
\begin{gathered}
T f(x)=\int_{\mathbb{R}^{n} \times \mathbb{R}^{n}} e^{i(x-y) \cdot \theta} b\left(x, y ; \theta^{\prime} ; \theta_{1}\right) f(y) d \theta d y, \\
b \in S^{p+\frac{1}{2}, l-\frac{1}{2}}\left(\mathbb{R}^{2 n} \times\left(\mathbb{R}^{n-1} \backslash 0\right) \times \mathbb{R}\right) .
\end{gathered}
$$

Note that on $\Lambda_{P} \backslash \Delta_{T^{*} \mathbb{R}^{n}}, T$ is a Fourier integral operator associated with $\Lambda_{P}$,

$$
T f(x)=\int_{\mathbb{R}^{n-1} \times \mathbb{R}^{n}} e^{i\left(x^{\prime}-y^{\prime}\right) \cdot \theta^{\prime}} c\left(x, y ; \theta^{\prime}\right) f(y) d \theta^{\prime} d y,
$$

where, for $x_{1} \neq y_{1}$,

$$
c\left(x, y ; \theta^{\prime}\right)=\int e^{i\left(x_{1}-y_{1}\right) \theta_{1}} b\left(x, y ; \theta^{\prime} ; \theta_{1}\right) d \theta_{1} \in S^{p+\frac{1}{2}}\left(\mathbb{R}^{2 n} \times\left(\mathbb{R}^{n-1} \backslash 0\right)\right) .
$$

Now, applying $T$ from (2.4) to $u(y)$, and applying stationary phase in $y, \xi_{1}, \theta^{\prime}$, we obtain, upon relabelling $\theta_{1}$ by $\xi_{1}$,

$$
T u(x)=\int_{\mathbb{R}^{n}} e^{i x \cdot \xi}\left[a\left(\left(0, x^{\prime}\right) ; \xi\right) b\left(x,\left(0, x^{\prime}\right) ; \xi^{\prime} ; \xi_{1}\right)+\ldots\right] d \xi .
$$

The amplitude in (2.7) is easily seen to belong to $S^{r+p+\frac{1}{2}-\frac{n}{4}, l-\frac{1}{2}}\left(\mathbb{R}^{n} \times\left(\mathbb{R}^{n-1} \backslash 0\right) \times \mathbb{R}\right)$; by $[G U, \S 1], T u \in I^{p^{\prime}, l^{\prime}}\left(\Lambda_{0}, \Lambda_{1}\right)$, with $p^{\prime}+l^{\prime}-\frac{n}{4}=r+p+\frac{1}{2}-\frac{n}{4}+l-\frac{1}{2}$ and $p^{\prime}+\frac{1}{2}-\frac{n}{4}=r+p+\frac{1}{2}-\frac{n}{4}$, so that $p^{\prime}=r+p, l^{\prime}=l$. 
To calculate the symbol of $T u$ on $\Lambda_{1} \backslash \Lambda_{0}$, we expand the amplitude $a$ in (2.7) about $\xi_{1}=0$ :

$$
T u(x)=\int_{\mathbb{R}^{n}} e^{i x \cdot \xi}\left[a\left(\left(0, x^{\prime}\right) ;\left(0, \xi^{\prime}\right)\right) b\left(x,\left(0, x^{\prime}\right) ; \xi^{\prime} ; \xi_{1}\right)+\xi_{1} d\left(x ; \xi^{\prime} ; \xi_{1}\right)\right] d \xi
$$

where $d\left(x ; \xi^{\prime} ; \xi_{1}\right) \in S^{r+p-\frac{1}{2}-\frac{n}{4}, l-\frac{3}{2}}\left(\mathbb{R}^{n} \times\left(\mathbb{R}^{n-1} \backslash 0\right) \times \mathbb{R}\right)$. The first term in (2.8) can be written as

$$
\int_{\mathbb{R}^{n-1}} e^{i x^{\prime} \cdot \xi^{\prime}} a\left(\left(0, x^{\prime}\right) ;\left(0, \xi^{\prime}\right)\right)\left[\int_{\mathbb{R}} e^{i x_{1} \xi_{1}} b\left(x,\left(0, x^{\prime}\right) ; \xi^{\prime} ; \xi_{1}\right) d \xi_{1}\right] d \xi^{\prime},
$$

which for $x_{1} \neq 0$ is an element of $I^{r+p}\left(\Lambda_{1}\right)$ ) with symbol as in (2.2); the second term is in $I^{r+p-1}\left(\Lambda_{1} \backslash \Lambda_{0}\right)$ and thus does not contribute to the principal symbol. Q.E.D.

Finally, we deal with the action of $I^{p, l}\left(\Delta_{T^{*} X}, \Lambda_{p}\right)$ on the double intersection class $I^{p^{\prime}, l^{\prime}}\left(\Lambda_{0}, \Lambda_{1}\right)$.

Proposition 2.2. Under the same assumptions as Proposition 2.1,

$$
T: I^{p^{\prime}, l^{\prime}}\left(\Lambda_{0}, \Lambda_{1}\right) \rightarrow I^{p+p^{\prime}+\frac{1}{2}, l+l^{\prime}-\frac{1}{2}}\left(\Lambda_{0}, \Lambda_{1}\right) .
$$

Thus, if $Q$ is a parametrix for $P(x, D)$,

$$
Q: I^{p^{\prime}, l^{\prime}}\left(\Lambda_{0}, \Lambda_{1}\right) \rightarrow I^{p^{\prime}+1-m, l^{\prime}-1}\left(\Lambda_{0}, \Lambda_{1}\right)
$$

Proof. We argue as in the proof of Proposition 2.1, but now $u \in I^{p^{\prime}, l^{\prime}}\left(\Lambda_{0}, \Lambda_{1}\right) \Rightarrow$

$$
u(x)=\int_{\mathbb{R}^{n}} e^{i x \cdot \xi} a\left(x ; \xi^{\prime} ; \xi_{1}\right) d \xi, \quad a \in S^{p^{\prime}+\frac{1}{2}-\frac{n}{4}, l^{\prime}-\frac{1}{2}}\left(\mathbb{R}^{n} \times\left(\mathbb{R}^{n-1} \backslash 0\right) \times \mathbb{R}\right),
$$

so that instead of (2.7) we have

$$
T u(x)=\int_{\mathbb{R}^{n}} e^{i x \cdot \xi} e\left(x ; \xi^{\prime} ; \xi_{1}\right) d \xi, \quad e \in S^{p+p^{\prime}+1-\frac{n}{4}, l+l^{\prime}-1}\left(\mathbb{R}^{n} \times\left(\mathbb{R}^{n-1} \backslash 0\right) \times \mathbb{R}\right)
$$

so that $T u \in I^{p+p^{\prime}+\frac{1}{2}, l+l^{\prime}-\frac{1}{2}}\left(\Lambda_{0}, \Lambda_{1}\right)$. For the parametrix $Q$, we specialize this to $p=\frac{1}{2}-m, l=-\frac{1}{2}$, yielding (2.10). Q.E.D.

Finally, we need

Proposition 2.3. Suppose $\Lambda_{1} \subset T^{*} X \backslash 0$ is a conic lagrangian which is characteristic for $P: \Lambda_{1} \subset \operatorname{char}(P)$. Then, if $T \in I^{p, l}\left(\Delta_{T^{*} X}, \Lambda_{P}\right)$,

$$
T: I^{r}\left(\Lambda_{1}\right) \rightarrow I^{r+p+\frac{1}{2}}\left(\Lambda_{1}\right)
$$

and thus

$$
Q: I^{r}\left(\Lambda_{1}\right) \rightarrow I^{r+1-m}\left(\Lambda_{1}\right) .
$$

Proof. Microlocalizing, we can find a $\Lambda_{0}$ such that $\left(\Lambda_{0}, \Lambda_{1}\right)$ are as in Proposition 2.2. For each $l^{\prime} \in \mathbb{R}, I^{r}\left(\Lambda_{1}\right) \subset I^{r, l^{\prime}}\left(\Lambda_{0}, \Lambda_{1}\right)$ ([GuU]), which is mapped by $T$ to $I^{r+p+\frac{1}{2}, l^{\prime}+l-\frac{1}{2}}\left(\Lambda_{0}, \Lambda_{1}\right)$ by (3.9). Intersecting over all $l^{\prime} \in \mathbb{R}$, we have $T: I^{r}\left(\Lambda_{1}\right) \rightarrow$ 
$\bigcap_{l^{\prime} \in \mathbb{R}} I^{r+p+\frac{1}{2}, l^{\prime}+l-\frac{1}{2}}\left(\Lambda_{0}, \Lambda_{1}\right)=I^{r+p+\frac{1}{2}}\left(\Lambda_{1}\right)$, again by the results of $[\mathrm{GuU}]$, proving (2.13). Since $Q \in I^{\frac{1}{2}-m,-\frac{1}{2}}\left(\Delta_{T * X}, \Lambda_{P}\right)$, (2.14) follows. Q.E.D.

\section{Plane Wave Ansatz for the Direct Problem}

We now analyze the approximate solution, $u_{0}+u_{1}$, to the direct problem described in the Introduction, under the assumption that the potential $q(x)$ is conormal to a smooth codimension $k$ submanifold. Let $S$ be given by a defining function,

$$
S=\left\{x \in \mathbb{R}^{n}: h(x)=0\right\},
$$

where $h \in C^{\infty}\left(\mathbb{R}^{n}, \mathbb{R}^{k}\right)$ satisfies $\operatorname{rank}(d h(x))=k$ for $x \in S$; in addition we assume $S$ has compact closure. Let

$$
q(x) \in I^{\mu}(S), \begin{cases}\mu<-\max \left(\left(1-\frac{2}{n}\right) k, k-1\right), & n \geqq 5 \\ \mu<-\max \left(\frac{k}{2}, k-1\right), & n=3 \text { or } 4\end{cases}
$$

be compactly supported and real-valued. Since, by (1.3), $q \in L^{p}\left(\mathbb{R}^{n}\right)$, for $p=\frac{n}{2}$ for $n \geqq 5$ and $p>2, n=3$ or 4 , it follows from a theorem of Phillips [P] that the scattering kernel $\alpha(s, \theta, \omega)$ of $q(x)$ exists; furthermore, the representation (3.34) below is valid.

Now define

$$
S_{1}=\left\{(x, t, \omega) \in \mathbb{R}^{n-1} \times S^{n-1}: x \in S\right\} ;
$$

regarding $q(x)$ as a distribution on $\mathbb{R}^{n-1} \times S^{n-1}$ independent of $t$ and $\omega$, one has

$$
q \in I^{\mu}\left(S_{1}\right) \text {. }
$$

We wish to find an approximation solution to the problem

$$
\left\{\begin{array}{l}
(\square+q(x)) u(x, t, \omega)=0 \quad \text { on } \mathbb{R}^{n-1} \times S^{n-1} \\
u(x, t, \omega)=\delta(t-x \cdot \omega), \quad t \ll 0,
\end{array}\right.
$$

where $\square=\frac{\partial^{2}}{\partial t^{2}}-\Delta_{\mathbb{R}^{n}}$ is the d'Alembertian on $\mathbb{R}^{n+1}$ acting independently of $\omega$. We look for an approximation

$$
u \sim u_{0}+u_{1}+\cdots+u_{j}+\cdots,
$$

where $u_{0}(x, t, \omega)=\delta(t-x \cdot \omega)$ and such that the series on the right is (formally) telescoping when $\square+q$ is applied. Thus $u_{j+1}=-\square^{-1}\left(q(x) u_{j}(x, t, \omega)\right)$, where $\square^{-1}$ is (say) the forward fundamental solution of $\square$. For the purposes of this paper, it suffices to consider the first two terms,

$$
u_{0}+u_{1}=\delta(t-x \cdot \omega)-\square^{-1}(q(x) \delta(t-x \cdot \omega)) .
$$


Now, the leading term in (3.6) is

$$
u_{0}(x, t, \omega)=\delta(t-x \cdot \omega) \in I^{0}\left(S_{+}\right)
$$

where

$$
S_{+}=\left\{(x, t, \omega) \in \mathbb{R}^{n+1} \times S^{n-1}: t-x \cdot \omega=0\right\} .
$$

The submanifolds $S_{+}$and $S_{1}$ intersect transversally; let $S_{2}=S_{+} \cap S_{1}$ be the resulting codimension $k+1$ submanifold at $\mathbb{R}^{n+1} \times S^{n-1}$. Let $\Lambda_{1}=N^{*} S_{1}$, $\Lambda_{+}=N^{*} S_{+}$and $\Lambda_{2}=N^{*} S_{2}$ be the respective conormal bundles, which, as described in Sect. 1, are lagrangian submanifolds of $T^{*}\left(\mathbb{R}^{n+1} \times S^{n-1}\right) \backslash 0$.

Proposition 3.1. a) $W F(q) \subset \Lambda_{1}$ and $W F\left(u_{0}\right) \subset \Lambda_{+}$.

b) $\Lambda$ and $\Lambda_{+}$are disjoint.

c) $\Lambda_{2}$ intersects $\Lambda_{1}$ and $\Lambda_{+}$cleanly in codimensions 1 and $k$, respectively, so that $\left(\Lambda_{1}, \Lambda_{2}\right)$ and $\left(\Lambda_{+}, \Lambda_{2}\right)$ are intersecting pairs.

Proof. a) Follows from (1.1).

b) $N_{(x, t, \omega)}^{*} S_{1}=\left\{\left(d h_{x}^{*}(\xi), 0,0\right): \xi \in \mathbb{R}^{k} \backslash 0\right\}$ while $N_{(x, t, \omega)}^{*} S_{+}=\left\{\left(-\sigma \omega, \sigma,-\sigma i_{\omega}^{*} x\right)\right.$ : $\sigma \in \mathbb{R} \backslash 0\}$, where $i_{\omega}: T_{\omega} S^{n-1} \subseteq T_{\omega} \mathbb{R}^{n}$.

c) Follows from the fact that $S_{2} \subset S_{1}$ is codimension 1 and $S_{2} \subset S_{+}$is codimension k. Q.E.D.

The second term in (3.6) is

$$
u_{1}=-\square^{-1}(q(x, t) \delta(t-x \cdot \omega)),
$$

where $\square^{-1}$ acts only in the $(x, t)$ variables. By Lemma (1.1), with $X=\mathbb{R}^{n+1} \times S^{n-1}$, $Y=S_{+}$and $Z=S_{1}$,

$$
q(x, t) \cdot \delta(t-x \cdot \omega) \in I^{0, \mu}\left(S_{+}, S_{2}\right)
$$

so that

$$
W F(q \cdot \delta) \subset \Lambda_{+} \cup \Lambda_{2} .
$$

To obtain $W F\left(u_{1}\right)$, recall that

$$
W F\left(\square^{-1} v\right) \subset\left(\Delta \cap \Lambda_{\square}\right) \circ W F(v), \quad \forall v \in \mathscr{E}^{\prime}\left(\mathbb{R}^{n+1} \times S^{n-1}\right),
$$

where $\Delta$ is the diagonal of $T^{*}\left(\mathbb{R}^{n+1} \times S^{n-1}\right) \backslash 0$ and $\Lambda_{\square}$ is the flowout of the characteristic variety

$$
\operatorname{char}(\square)=\left\{(x, t, \omega ; \xi, \tau, \Omega):|\tau|^{2}=|\xi|^{2}\right\}
$$

of $\square$ (acting on $\mathbb{R}^{n+1} \times S^{n-1}$ ). In (3.10), $\Delta \cup \Lambda_{\square}$ acts as a relation between subsets of $T^{*}\left(\mathbb{R}^{n+1} \times S^{n-1}\right) \backslash 0$; if course, $\Delta$ acts as the identity. Also, $\Lambda_{\square} \circ \Lambda_{+}=\Lambda_{+}$since $\Lambda_{+}$is characteristic for $\square$. Thus

$$
W F\left(u_{1}\right) \subset \Lambda_{+} \cup \Lambda_{2} \cup \Lambda_{\square} \circ \Lambda_{2} .
$$

To understand the last term in (3.12), note that $\Lambda_{2}$ is a $(k+1)$-plane over $S_{2}$ : $\Lambda_{2}=\left\{\left(x, x \cdot \omega, \omega ; v-\tau \omega, \tau,-\tau i_{\omega}^{*} x\right): x \in S, \omega \in S^{n-1},(v, \tau) \in\left(N_{x}^{*} S \times \mathbb{R}\right) \backslash 0\right\}$, 
where $i_{\omega}^{*}$ denotes the restriction of an element of $\mathbb{R}^{n^{*}}$ to $T_{\omega} S^{n-1}$. The intersection of $\Lambda_{2}$ with char $(\square)$ in these coordinates is given by

$$
\Sigma=\Lambda_{2} \cap \operatorname{char}(\square)=\{v \cdot(v-2 \tau \omega)=0\} .
$$

Above a point $(x, x \cdot \omega, \omega) \in S_{2}$ such that $N_{x}^{*} S \subset \omega^{\perp}$, the fiber of $\Lambda_{2} \cap \operatorname{char}(\square)$ is just $\left\{\left(-\tau \omega, \tau,-\tau i_{\omega}^{*} x\right): \tau \in \mathbb{R} \backslash 0\right\}$, while if $N_{x}^{*} S \neq \omega^{\perp}$, the fiber is a smooth $k$-dimensional cone in $T_{(x, x \cdot \omega, \omega)}^{*} \mathbb{R}^{n+1} \times S^{n-1} \backslash 0$. Since $N_{x}^{*} S \subset \omega^{\perp}$ iff $\omega \in T_{x} S$, we see that the degenerate points correspond to the incoming plane wave being tangent to $S$. Let

$$
S_{3}=\left\{(x, x \cdot \omega, \omega) \in S_{2}: \omega \in T_{x} S\right\}
$$

then $S_{3} \subset S_{2}$ is codimension $k$ and away from $\Sigma_{3}=\left.\Lambda_{2}\right|_{S_{3}}, \Sigma$ is a smooth hypersurface in $\Lambda_{2}$.

To discuss the geometry further, we first consider the situation when $S$ is a hypersurface $(k=1)$, so that $h(x)$ is scalar-valued with gradient $h_{x}$, and (3.13) may be rewritten as

$$
\Lambda_{2}=\left\{\left(x, x \cdot \omega, \omega ; \lambda h_{x}-\tau \omega, \tau,-\tau i_{\omega}^{*} x\right) ; x \in S,(\lambda, \tau) \in \mathbb{R}^{2} \backslash 0\right\} .
$$

In these coordinates,

$$
\Lambda_{2} \cap \operatorname{char}(\square)=\left\{\lambda\left(h_{x}^{2} \lambda-2\left(\omega \cdot h_{x}\right) \tau\right)=0\right\},
$$

and thus $\Sigma=\Sigma_{+} \cup \Sigma_{-}$, where $\Sigma_{+}=\{\lambda=0\}$ and $\Sigma_{-}=\left\{h_{x}^{2} \lambda-2\left(\omega \cdot h_{x}\right) \tau=0\right\}$ are smooth hypersurfaces intersecting transversally over $S_{3}=\left\{\omega \cdot h_{x}=0\right\}$. Note that $\Sigma_{+}=\Lambda_{+} \cap \Lambda_{2}$ and thus the flowout $\Lambda_{\square}{ }^{\circ}\left(\Sigma_{+} \backslash \Sigma_{3}\right)$ of $\Sigma_{+} \backslash \Sigma_{3}$ by the Hamiltonian vector field

$$
H_{\square}=-\xi \cdot \frac{\partial}{\partial \xi}+\tau \frac{\partial}{\partial \tau}
$$

is contained in $\Lambda_{+}$. On the other hand, the flowout of $\Sigma_{-} \backslash \Sigma_{3}$ is a new lagrangian, which we denote by $\Lambda_{-}$. In fact, assuming, as we may, that $h_{x}^{2} \equiv 1$,

$$
\Sigma_{-}=\left\{\left(y, y \cdot \omega, \omega ; \tau v(y, \omega), \tau,-\tau i_{\omega}^{*} y\right): y \in S, \omega \in S^{n-1}, \tau \in \mathbb{R} \backslash 0\right\},
$$

where $v(y, \omega)=2\left(\omega \cdot h_{y}\right) h_{y}-\omega$. Note that $v^{2}=1$ and $v(y, \omega)=-\omega$ iff $\omega \cdot h_{y}=0$, i.e, only at $\Sigma_{3}$. $H_{\square}$ can only be tangent to $\Sigma_{-}$if it arises as the image of a vector $Y \cdot \frac{\partial}{\partial y}$ under the diferential of the parametrization (3.17); but then $Y=-\xi=-\tau v(y, \omega)$ and $\omega \cdot Y=\tau$, which imply that $v(y, \omega)=-\omega$, which only occurs at $\Sigma_{3}$ by the above comment. Thus, $H_{\square} \phi \mid \Sigma_{-}$on $\Sigma_{-} \backslash \Sigma_{3}$, and $\Lambda_{-}=\Lambda_{\square} \circ\left(\Sigma_{-} \backslash \Sigma_{3}\right)$ is a smooth lagrangian, intersecting $\Lambda_{2}$ cleanly in codimension 1. Explicitly,

$$
\begin{aligned}
\Lambda_{-}= & \left(y-r v(y, \omega), y \cdot \omega+r, \omega ; \tau v(y, \omega), \tau,-\tau i_{\omega}^{*} y\right): y \in S, \omega \in S^{n-1}, \omega \cdot h_{y} \neq 0, \\
& r \in \mathbb{R}, \tau \in \mathbb{R} \backslash 0\} .
\end{aligned}
$$


We remark here that $\Lambda_{-}$is the conormal bundle of a smooth hypersurface, which we denote by $S_{-}$. In fact, the differential of the projection $\pi$ from $\Lambda_{-}$onto the spatial variables is

$$
\frac{D(x, t, \omega)}{D(y, r, \omega, \tau)}=\left(\begin{array}{cccc}
j-r j^{*} d_{y} v & -v & -r d_{\omega} v & 0 \\
j^{*} \omega & 1 & i_{\omega}^{*} x & 0 \\
0 & 0 & I & 0
\end{array}\right),
$$

where $j$ denotes the differential of the inclusion $S \subseteq \mathbb{R}^{n}$, from which we see that

$$
\operatorname{rank}(d \pi)=n+\operatorname{rank}\left(j-r j^{*} d_{y} v+j^{*} \omega \otimes v\right) .
$$

Away from $S_{3}, v(y, \omega) \neq-\omega$ and the rank of the second term in (3.19) is $n-1$; thus rank $(d \pi)=2 n-1$ and $\Lambda_{-}=N^{*} S_{-}$, for $S_{-} \subset \mathbb{R}^{n+1} \times S^{n-1}$ a smooth hypersurface.

Now consider the case when the codimension of $S$ satisfies $1<k<n$. Then $\Sigma$, defined by (3.14), has a conical singularity at $\Sigma_{3}$. We will actually work away from a larger set,

$$
\begin{aligned}
\Sigma_{2}= & \left\{\left(y, y \cdot \omega, \omega ; v-\tau \omega, \tau,-\tau i_{\omega}^{*} y\right):(y, v) \in N^{*} S, \omega \in S^{n-1},\right. \\
& \tau \in \mathbb{R} \backslash 0, v \cdot \omega=0\} .
\end{aligned}
$$

By the same reasoning as for $k=1, H_{\square} \pitchfork \Sigma$ on $\Sigma \backslash \Sigma_{2}$; thus, $\Lambda_{-}$, which we define in this case to be $\Lambda_{\square}{ }^{\circ}\left(\Sigma \backslash \Sigma_{2}\right)$, is a smooth lagrangian intersecting $\Lambda_{2}$ cleanly in codimension 1. Furthermore, since $\Lambda_{+} \cap \Lambda_{2} \subset \Sigma_{2}, \Lambda_{+}$and $\Lambda_{-}$are disjoint (although $\Lambda_{+} \cap \bar{\Lambda}_{-} \neq \emptyset$.) We can parametrize $\Sigma \backslash \Sigma_{2}$ and $\Lambda_{-}$by solving $v \cdot(v-2 \tau \omega)$ for $\tau$, which we can do away from $\Sigma_{2}: \tau=\tau(y, v, \omega)=\frac{v^{2}}{2(v \cdot \omega)}$. Note that $|v-\tau \omega|=|\tau|$. Thus,

$$
\Sigma \backslash \Sigma_{2}=\left\{\left(y, y \cdot \omega, \omega ; v-\tau \omega, \tau,-\tau i_{\omega}^{*} y\right):(y, v) \in N^{*} S \backslash 0, \omega \in S^{n-1}, v \cdot \omega \neq 0\right\}
$$

and

$$
\begin{aligned}
\Lambda_{-}= & \left\{\left(y-r w(y, v, \omega), y \cdot \omega, \omega ; v-\tau \omega, \tau,-\tau i_{\omega}^{*} y\right):(y, v) \in N^{*} S \backslash 0, \omega \in S^{n-1},\right. \\
& r \in \mathbb{R}, v \cdot \omega \neq 0\},
\end{aligned}
$$

where $w(y, v, \omega)=\frac{v}{\tau}-\omega$; note that $w^{2}=1$.

When $k=n$, so that $S$ is a finite set of points in $\mathbb{R}^{n}$, introducing $\Sigma_{3}$ or $\Sigma_{2}$ is unnecessary: $\Sigma$ is smooth, as is $\Lambda_{-}=\Lambda_{\square} \circ \Sigma . \Lambda_{+}$and $\Lambda_{-}$intersect, however, and we will work away from this intersection by using the parametrization (3.22).

Finally, we note that for $1<k \leq n, \Lambda_{-}$is contained in the conormal bundle of a submanifold of $\mathbb{R}^{n+1} \times S^{n-1}$ having a conical singularity along $S_{2}$. Not needing this fact below, we will not describe its structure further.

Returning now to the second term, $u_{1}(x, t, \omega)$, of the approximate solution for the direct problem, let $\mathcal{O}^{\prime} \Subset \mathcal{O} \subset \Lambda_{2}$ be conic neighborhoods of $\Sigma_{3}, \Sigma_{2}$ or $\left(\Lambda_{+} \cap \Lambda_{2}\right) \cap \Sigma$ in the cases when $k=1,1<k<n$ or $k=n$, respectively. Let 
$L=\Lambda_{\square} \circ \mathcal{O}, L^{\prime}=\Lambda_{\square} \circ \mathcal{O}^{\prime}$ be the conic neighborhoods of $\mathcal{O}, \mathcal{O}^{\prime}$ in $T^{*}\left(\mathbb{R}^{n+1} \times S^{n-1}\right)$ invariant under the Hamiltonian flow. Then (3.12) becomes

$$
W F\left(u_{1}\right) \backslash L \subset \Lambda_{+} \cup \Lambda_{2} \cup \Lambda_{-} .
$$

In fact, microlocalizing $u_{1}$ away from $L, u_{1}$ belongs to the sum of the spaces of lagrangian distributions associated with the pairs $\left(\Lambda_{2}, \Lambda_{+}\right)$and $\left(\Lambda_{2}, \Lambda_{-}\right)$. Let $\chi(x, t, \omega, \xi, \tau, \Omega) \in C^{\infty}\left(T^{*}\left(\mathbb{R}^{n+1} \times S^{n-1}\right) \backslash 0\right)$ be homogeneous of degree 0 in the fiber variables, with $\chi \equiv 0$ on $L^{\prime}$ and $\chi \equiv 1$ on $L^{c}$. The corresponding pseudodifferential operator $\chi\left(x, t, \omega, D_{x}, D_{t}, D_{\omega}\right)$ has the property that $\left[\square^{-1}, \chi\right]$ is microlocally supported on the annulus $L \cap L^{\prime c}$. Let

$$
\tilde{u}_{1}=-\square^{-1}(\chi(q \cdot \delta)) ;
$$

then $\tilde{u}_{1}-u_{1}$ is microlocally smooth on $L^{c}$ by the above comment. Translating (3.8) from conormal to lagrangian language, we find that

$$
q(x) \cdot \delta(t-x \cdot \omega) \in I^{\frac{1-n}{2}, \mu+\frac{k}{2}}\left(\Lambda_{2}, \Lambda_{+}\right) .
$$

The same is true for $\chi(q \cdot \delta)$, and since, by [GuU], the double intersection spaces microlocalize, one has $\chi(q \cdot \delta)=u^{+}+u^{-}$, where

$$
\left\{\begin{array}{l}
u^{+} \in I^{\frac{1-n}{2}, \mu+\frac{k}{2}}\left(\Lambda_{2} \backslash L, \Lambda_{+} \backslash L\right) \\
u^{-} \in I^{\mu+\frac{k+1-n}{2}}\left(\Lambda_{2} \backslash L\right)
\end{array}\right.
$$

with $u^{ \pm}$supported microlocally near $\Lambda_{2} \cap \Lambda_{ \pm}$. Applying $-\square^{-1} \in$ $I^{-\frac{3}{2},-\frac{1}{2}}\left(\Lambda_{T^{*}\left(\mathbb{R}^{n+1} \times S^{n-1}\right)}, \Lambda_{\square}\right)$ to $u^{+}$and $u^{-}$, and using Propositions 2.2 and 2.1, respectively, we find that

$$
\left\{\begin{array}{l}
-\square^{-1}\left(u^{+}\right) \in I^{-\frac{(n+1)}{2}, \mu+\frac{k}{2}-1}\left(\Lambda_{2} \backslash L, \Lambda_{+} \backslash L\right) \\
-\square^{-1}\left(u^{-}\right) \in I^{\mu+\frac{k-2-n}{2},-\frac{1}{2}}\left(\Lambda_{2} \backslash L, \Lambda_{-} \backslash L\right) .
\end{array}\right.
$$

Since the variable $t$ is bounded on $\Lambda_{2}$, by (1.4) we have

$$
u_{1} \in I^{-\frac{(n+1)}{2}}\left(\Lambda_{+} \backslash L\right)+I^{\mu+\frac{k-2-n}{2}}\left(\Lambda_{-} \backslash L\right), \quad t \gg 0,
$$

and thus the approximate solution

$$
u_{0}+u_{1} \in I^{-\frac{(n-1)}{2}}\left(\Lambda_{+} \backslash L\right)+I^{\mu+\frac{k-2-n}{2}}\left(\Lambda_{-} \backslash L\right), \quad t \gg 0,
$$

Furthermore, the analysis shows that $W F\left(u_{1}\right) \subset \Lambda_{\square} \circ \Lambda_{2}$.

Now let $R: \mathscr{E}^{\prime}\left(\mathbb{R}^{n}\right) \rightarrow \mathscr{E}^{\prime}\left(\mathbb{R} \times S^{n-1}\right)$ be the Radon transform

$$
(R f)(s, \theta)=\int_{x \cdot \theta=s} f(x) d \sigma(x),
$$

where $d \sigma$ is normalized Lebesgue measure on the hyperplane $\{x \cdot \theta=s\}$. Acting in the $x$ variable, $R$ is defined on those elements of $\mathscr{D}^{\prime}\left(\mathbb{R}^{n} \times \mathbb{R} \times S^{n-1}\right)$ having compact support in $x$ for each $t, \omega ; R$ is an elliptic Fourier integral operator, $R \in I^{(1-n) / 2}\left(C_{R}\right)$, 
where $C_{R}$ is the local canonical graph $\subset T^{*}\left(\mathbb{R} \times S^{n-1} \times \mathbb{R} \times S^{n-1} \times \mathbb{R}^{n} \times \mathbb{R} \times S^{n-1}\right)$ given by

$$
\begin{aligned}
& C_{R}=\left\{\left(x \cdot \theta, \theta, t, \omega, \sigma,-\sigma i_{\theta}^{*}(x), \tau, \Omega ; x, t, \omega, \sigma \theta, \tau, \Omega\right):\right. \\
&\left.(x, t, \omega) \in \mathbb{R}^{n+1} \times S^{n-1}, \theta \in S^{n-1}, \sigma \in \mathbb{R} \backslash 0, \tau \in \mathbb{R}, \Omega \in T_{\omega}^{*} S^{n-1}\right\} .
\end{aligned}
$$

The modified (Lax-Phillips) Radon transform [LP], which maps $\mathbb{C}^{2}$ - to $\mathbb{C}$-valued distributions, is defined by

$$
R_{\mathrm{LP}}\left(\begin{array}{c}
v_{0} \\
v_{1}
\end{array}\right)=C_{n} D_{s}^{\frac{n-1}{2}}\left(D_{s} R v_{0}-R v_{1}\right), \quad n \text { odd } .
$$

For $n$ even one replaces in (3.32) $D_{s}^{\frac{n-1}{2}}$ by $\left|D_{s}^{\frac{n-1}{2}}\right|$. For another discussion of the time dependent approach to scattering theory and also for a more detailed treatment of the odd dimensional case, see [Pe].

The scattering kernel can be expressed in terms of $R_{\mathrm{LP}}$ and the solution to the direct problem (3.5) as follows [MU2]: letting

$$
w=D_{t}^{\frac{n-3}{2}}(u(x, t, \omega)-\delta(t-x \cdot \omega)), \quad n \text { odd },
$$

one has the relation

$$
\alpha(t-s, \theta, \omega)=\delta(t-s) \otimes \delta(\theta-\omega)+R_{\mathrm{LP}}\left(\begin{array}{c}
w \\
D_{t} \omega
\end{array}\right), \quad t \gg 0 .
$$

For $n$ even one replaces $D_{t}$ in (3.33) by $\left|D_{t}\right|$.

Now, acting on the argument $u-\delta, w$ and $D_{t} w$ are pseudodifferential operators of orders $\frac{n-3}{2}$ and $\frac{n-1}{2}$, respectively, and thus

$$
(\alpha-\delta \otimes \delta)(\tau-s, \theta, \omega)=F(u-\delta),
$$

where $F \in I^{\frac{n-1}{2}}\left(C_{R}\right)$. Furthermore, if we denote the fiber variables in $T^{*}\left(\mathbb{R} \times S^{n-1} \times \mathbb{R} \times S^{n-1}\right)$ and $T^{*}\left(\mathbb{R}^{n+1} \times S^{n-1}\right)$ by $(\sigma, \Theta, \tau, \Omega)$ and $\left(\xi, \tau^{\prime}, \Omega^{\prime}\right)$, respectively, the symbol of $F$ is an elliptic factor times $\sigma-\tau^{\prime}$. This is elliptic on the region of $C_{R}$ giving rise to the first components in $(3.40)_{1}$ and $(3.40)_{k}$, which are shown below to be those of interest.

To deal with the translation-invariance in (3.35), we introduce, for $t_{0} \gg 0$, the mapping $\rho: \mathbb{R} \times S^{n-1} \times S^{n-1} \rightarrow \mathbb{R} \times S^{n-1} \times \mathbb{R} \times S^{n-1}, \rho(s, \theta, \omega)=\left(t_{0}+s, \theta, t_{0}, \omega\right)$, which induces a restriction mapping

$$
\rho^{*}: \mathscr{D}_{\rho}^{\prime}\left(\mathbb{R} \times S^{n-1} \times \mathbb{R} \times S^{n-1}\right) \rightarrow \mathscr{D}^{\prime}\left(\mathbb{R} \times S^{n-1} \times S^{n-1}\right) .
$$

Here, $\mathscr{D}_{\rho}^{\prime}$ denotes those distributions whose wavefront sets are disjoint from the normals of $\rho . \rho^{*}$ is a Fourier integral operator, $\rho^{*} \in I^{\frac{1}{4}}\left(C_{\rho}\right)$, where

$$
\begin{aligned}
C_{\rho}= & \left\{\left(s, \theta, \omega, \sigma, \Theta, \Omega ; t_{0}+s ; \theta, t_{0}, \omega, \sigma, \Theta, \eta, \Omega\right):\right. \\
& \left.s \in \mathbb{R}, \theta, \omega \in S^{n-1},(\sigma, \Theta, \tau, \Omega) \in T^{*}\left(\mathbb{R} \times S^{n-1} \times S^{n-1}\right) \backslash 0\right\} .
\end{aligned}
$$

Thus, the scattering kernel can be expressed by means of

$$
\left(\alpha-\delta_{0} \otimes \delta\right)(s, \theta, \omega)=\rho^{*} F(u-\delta) .
$$


We first examine the right side of (3.37) applied to the approximate solution $u_{0}+u_{1}$; the comparison with $\rho^{*} F(u-\delta)$ will be made later. Of course, $\rho^{*} F\left(u_{0}+u_{1}-\delta\right)=\rho^{*} F\left(u_{1}\right)$. Since $C_{R}$ is a local canonical graph, by (3.28) we have

$$
F\left(u_{1}\right) \in I^{-1}\left(C_{R^{\circ}} \Lambda_{+}\right)+I^{\mu+\frac{k-3}{2}}\left(C_{R^{\circ}} \Lambda_{-}\right) \text {. }
$$

Applying (3.31) to $\Lambda_{+}$, we see that $C_{R^{\circ}} \Lambda_{+}$has two components,

$$
\begin{aligned}
C_{R} \circ \Lambda_{+}= & \left\{\left(y \cdot \omega, \omega, y \cdot \omega, \omega ; \sigma,-\sigma i_{\omega}^{*} y,-\sigma, \sigma i_{\omega}^{*} y\right): y \in \mathbb{R}^{n}, \omega \in S^{n-1}, \sigma \in \mathbb{R} \backslash 0\right\} \\
& \cup\left[-y \cdot \omega,-\omega, y \cdot \omega, \omega ; \sigma,-\sigma i_{-\omega}^{*} y, \sigma,-\sigma i_{\omega}^{*} y\right): y \in \mathbb{R}^{n}, \\
& \left.\omega \in S^{n-1}, \sigma \in \mathbb{R} \backslash 0\right\} .
\end{aligned}
$$

Similarly, applying $C_{R}$ to $\Lambda_{-}$in the cases $k=1$ and $1<k \leqq n$, we obtain

$$
\begin{aligned}
C_{R^{\circ}} \Lambda_{-}= & \left(r-y \cdot v(y, \omega),-v(y, \omega), y \cdot \omega+r, \omega ; \sigma,-\sigma i_{-}^{*} y,-\sigma, \sigma i_{\omega}^{*} y\right): \\
& \left.y \in S, \omega \in S^{n-1}, r \in \mathbb{R}, \sigma \in \mathbb{R} \backslash 0\right\} \\
\cup & \left\{\left(y \cdot v(y, \omega)-r, v(y, \omega), y \cdot \omega+r, \omega ; \sigma,-\sigma i_{v}^{*} y, \sigma,-\sigma i_{\omega}^{*} y\right):\right. \\
& \left.y \in S, \omega \in S^{n-1}, r \in \mathbb{R}, \sigma \in \mathbb{R} \backslash 0\right\}, \\
C_{R} \circ \Lambda_{-}= & \left\{\left(r-y \cdot w(y, v, \omega),-w(y, v, \omega), y \cdot \omega+r, \omega ;-\tau(y, v, \omega), \tau i_{-}^{*}{ }_{w} y, \tau,\right.\right. \\
& \left.\left.-\tau i_{\omega}^{*} y\right):(y, v) \in N^{*} S \backslash 0, \omega \in S^{n-1}, v \cdot \omega \neq 0, r \in \mathbb{R}\right\} \\
\cup & \left\{\left(y \cdot w(y, v, \omega)-r, w(y, v, \omega), y \cdot \omega+r, \omega ; \tau(y, v, \omega),-\tau i_{\omega}^{*} y, \tau,\right.\right. \\
& \left.\left.-\tau i_{\omega}^{*} y\right):(y, v) \in N^{*} S \backslash 0, \omega \in S^{n-1}, v \cdot \omega \neq 0, r \in \mathbb{R}\right\},
\end{aligned}
$$

respectively. We also note that

$$
\begin{aligned}
C_{R} \circ \Lambda_{1}= & \left\{\left( \pm \frac{v}{|v|} \cdot y, \pm \frac{v}{|v|}, \tau, \omega ; \pm|v|, \pm-|v| i_{ \pm}^{*} \frac{v}{|v|} y, 0,0\right):\right. \\
& \left.(y, v) \in N^{*} S \backslash 0, \tau \in \mathbb{R}, \omega \in S^{n-1}\right\} .
\end{aligned}
$$

Since it is impossible for both $s$ and $t$ to be $>0$ on the second component of $C_{R} \circ \Lambda_{+}$in (3.39) and the second component of $C_{R} \circ \Lambda_{-}$in $(3.40)_{1},(3.40)_{k}$, the application of $C_{\rho}$ to these components is empty. Define the peak lagrangian

$$
\begin{aligned}
\hat{\Lambda}_{+}=C_{\rho} \circ C_{R^{\circ}} \Lambda_{+} & =\left\{\left(0, \omega, \omega ; \sigma,-\sigma i_{\omega}^{*} y, \sigma_{\omega}^{*} y\right): \omega \in S^{n-1}, y \in \mathbb{R}^{n}, \sigma \in \mathbb{R} \backslash 0\right\} \\
& \subseteq\left\{(0, \omega, \omega ; \sigma,-\Omega, \Omega):(\omega, \Omega) \in T^{*} S^{n-1}, \sigma \in \mathbb{R} \backslash 0\right\} \\
& =N^{*}\{s=0, \theta=\omega\}
\end{aligned}
$$

and the reflected lagrangian,

$$
\begin{aligned}
& \hat{\Lambda}_{-}= C_{\rho} \circ C_{R} \circ \Lambda_{-}=\left\{\left(-y \cdot(v(y, \omega)+\omega), v(y, \omega), \omega ; \sigma,-\sigma i_{-}^{*} y, \sigma i_{\omega}^{*} y\right):\right. \\
&\left.y \in S, \omega \in S^{n-1}, \sigma \in \mathbb{R} \backslash 0\right\}, \\
& \hat{\Lambda}_{-}=\left\{\left(-y \cdot(w(y, v, \omega)+\omega),-w(y, v, \omega), \omega ; \tau(y, v, \omega),-\tau i_{-w}^{*} y, \tau i_{\omega}^{*} y\right):\right. \\
&\left.(y, v) \in N^{*} S \backslash 0, \omega \in S^{n-1}\right\},
\end{aligned}
$$


for $k=1$ and $1<k \leqq n$, respectively. One computes easily that the application of

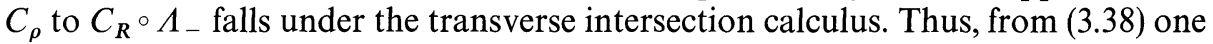
obtains

$$
\rho_{*} F\left(u_{1}\right) \in I^{-\frac{3}{4}}\left(\hat{\Lambda}_{+}\right)+I^{\mu+\frac{k}{2}-\frac{5}{4}}\left(\hat{\Lambda}_{-}\right) .
$$

To compare this with the true scattering kernel given by (3.35), set $\bar{u}=u-\left(u_{0}+u_{1}\right)$. Then $\bar{u} \equiv 0$ for $t \gg 0$ and

$$
\begin{aligned}
(\square+q) \bar{u} & =(\square+q) u-(\square+q)\left(u_{0}+u_{1}\right) \\
& =0-\square u_{0}-q \cdot u_{0}-\square u_{1}-q \cdot u_{1} \\
& =-q \cdot u_{1} .
\end{aligned}
$$

Some care needs to be taken in interpreting the product $q \cdot u_{1}$. Let $M_{q}$ denote multiplication by $q$; we will show that $M_{q}$ is a "pseudodifferential operator with singular symbol" on $\mathbb{R}^{n+1} \times S^{n-1}$. In fact, the Schwartz kernel of $M_{q}$ is

$$
K_{M_{q}}\left((x, \tau, \omega),\left(x^{\prime}, \tau^{\prime}, \omega^{\prime}\right)\right)=q(x) \delta\left(x-x^{\prime}\right) \delta\left(t-t^{\prime}\right) \delta\left(\omega-\omega^{\prime}\right),
$$

which belongs to $I^{\mu+\frac{k}{2},-\left(\mu+\frac{k}{2}\right)}\left(\Delta_{T^{*}\left(\mathbb{R}^{n-1} \times S^{n-1}\right)}, \Lambda_{S}^{\prime}\right)$, where $\Lambda_{S}$ is the flowout of $\left.T^{*}\left(\mathbb{R}^{n+1} \times S^{n-1}\right)\right|_{S}=\{(x, \tau, \omega, \xi, \tau, \Omega): x \in S\}$,

$$
\begin{aligned}
\Lambda_{S}= & \left\{(x, \tau, \omega, \xi+\eta, \tau, \Omega ; x, \tau, \omega, \xi, \tau, \Omega):\left.(x, \tau, \omega, \xi, \tau, \Omega) \in T^{*}\left(\mathbb{R}^{n+1} \times S^{n-1}\right)\right|_{S},\right. \\
& \left.\eta \in N_{x}^{*} S\right\} .
\end{aligned}
$$

The resulting operator is defined when acting on distributions $v \in D^{\prime}\left(R^{n+1} \times S^{n-1}\right)$ such that $\Lambda_{S} \circ W F(v) \cap(0)=\phi$, where (0) is the 0 -section. From (3.46), we see that $M_{q} v$ is defined for $v$ such that $W F(v) \cap \Lambda_{1}=\phi$. If $K \subset T^{*}\left(R^{n+1} \times S^{n-1}\right) \backslash(0)$ is a closed, conic set disjoint from $\Lambda_{1}$, and $H_{K}^{s}$ consists of those elements of the local Sobolev space $H_{\mathrm{loc}}^{s}$ with wave front set contained in $K$, it follows from the results of $[\mathrm{GU}]$ that

$$
M_{q}: H_{K}^{s} \rightarrow H_{\mathrm{loc}}^{s-(\mu+k)+} .
$$

To deal with $v$ 's with wavefront set near $\Lambda_{1}$, we make use of the results of Sect. 1. Introduce zeroth order pseudodifferential operators $\chi_{j}(D)=$ $\chi_{j}\left(x, t, \omega, D_{x}, D_{t}, D_{\omega}\right), 1 \leqq j \leqq 4$, on $R^{n+1} \times S^{n-1}$, forming a microlocal partition of unity, such that $\chi_{1}(D)$ is supported on a neighborhood ${ }^{c} K$ of $\Lambda_{1}$, where $K$ is as above; $\chi_{2}(D)$ is supported near $\left(\Lambda_{+} \cup \Lambda_{-}\right) \backslash L ; \chi_{3}(D)$ is supported near $L$; and $\chi_{4}(D)$ is such that $\sum_{j=1}^{4} \chi_{j}(D)=I$. Now, since $q(x) \delta(t-x \cdot \omega) \in I^{\mu+\frac{k+1-n}{2}}\left(\Lambda_{2}\right)$ away from $\Lambda_{+}$,

$$
\chi_{1}(D) u_{1}=-\chi_{1}(D) \square^{-1}(q \delta) \in I^{\mu+\frac{k-3-n}{2}}\left(\Lambda_{2}\right)=I^{\mu-2}\left(S_{2}\right)
$$

and is supported near $\Lambda_{1}$. By Lemma 1.3,

$$
M_{q} \chi_{1} u_{1} \in I^{\mu, \mu+k-2}\left(S_{1}, S_{2}\right)=I^{2 \mu+\frac{3 k-3-n}{2},-\mu+\frac{3-k}{2}}\left(\Lambda_{1}, \Lambda_{2}\right),
$$

since $\mu-2<-k, 2 \mu-2<-k$. By (3.27),

$$
\begin{gathered}
\chi_{2}(D) u_{1} \in I^{-\frac{n+1}{2}, \mu+\frac{k-2}{2}}\left(\Lambda_{2}, \Lambda_{+}\right)+I^{\mu+\frac{k-2-n}{2},-\frac{1}{2}}\left(\Lambda_{2}, \Lambda_{-}\right) \\
=I^{-\frac{k+1}{2}, \mu+\frac{k-3}{2}}\left(S_{+}, S_{2}\right)+I^{\mu-1,-1}\left(S_{-}, S_{2}\right) .
\end{gathered}
$$


Applying Lemma 1.5 with $Y_{1}=S_{1}, Y_{2}=S_{2}$ and $Y_{+}=S_{+}$or $S_{-}$, we obtain

$$
\begin{aligned}
M_{q} \chi_{2} u_{1} \in I^{-\frac{k+1}{2}, \mu+\frac{k-3}{2}+(\mu+k)_{+, \varepsilon}}\left(S_{+}, S_{2}\right)+I^{-\frac{k+1}{2}+\left(2 \mu+\frac{3 k-3}{2}\right)_{+, \varepsilon}, 0}\left(S_{+}, S_{2}\right) \\
+I^{\mu-1,-1+(\mu+k)_{+, \varepsilon}}\left(S_{-}, S_{2}\right)+I^{\mu-1+(\mu-1+k)_{+, \varepsilon}, 0}\left(S_{-}, S_{2}\right) \\
+I^{\mu,-\frac{k+1}{2}+\left(\mu+\frac{3 k-3}{2}\right)_{+, \varepsilon}}\left(S_{1}, S_{2}\right)+I^{\mu, \mu-1+(-1+k)_{+, \varepsilon}}\left(S_{1}, S_{2}\right),
\end{aligned}
$$

where $(x+k)_{+, \varepsilon}=(x+k)_{+}+\varepsilon \delta_{x,-k}$ for any $\varepsilon>0$.

Since $\chi_{3}(D) u_{1} \in H_{K}^{s_{0}}$ for some $s_{0} \in R, M_{q} \chi_{2} u_{1} \in H_{\mathrm{loc}}^{s_{0}-(\mu+k)+}$ by (3.47), and $W F\left(M_{q} \chi_{2} u_{1}\right) \subset L$ by (3.46) and the definition of $L$. Finally, $M_{q} \chi_{4} u_{1} \in$ $I^{-k+(\mu+k)+, \varepsilon}\left(S_{1}, S_{2}\right)$; this latter space also contains $M_{q} \chi_{1} u_{1}$, since $-k+(\mu+k)_{+, \varepsilon} \geqq \mu$.

Having described the right side of (3.45), we now apply the forward fundamental solution $\square^{-1}$ to both sides. Since $I^{M, M^{\prime}}\left(S_{ \pm}, S_{2}\right)=I^{M+\frac{k-n}{2}, M^{\prime}+\frac{1}{2}}\left(\Lambda_{2}, \Lambda_{ \pm}\right)$, (2.10) implies that

$$
\square^{-1}: I^{M, M^{\prime}}\left(S_{ \pm}, S_{2}\right) \rightarrow I^{M-1, M^{\prime}-1}\left(S_{ \pm}, S_{2}\right) .
$$

Furthermore, $\square^{-1}$ acts on $I^{M, M^{\prime}}\left(S_{1}, S_{2}\right)$ as a pseudodifferential operator of order -2. Finally, $\square^{-1}: H^{s} \rightarrow H_{\text {loc }}^{s+1}$. Thus, (3.45) becomes

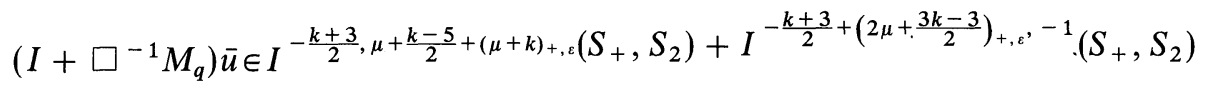

$$
\begin{aligned}
& +I^{\mu-2,-2+(\mu+k)_{+, \varepsilon}}\left(S_{-}, S_{2}\right)+I^{\mu-2+(\mu-1+k)_{+, \varepsilon},-1}\left(S_{-}, S_{2}\right) \\
& +I^{\mu-2,-\frac{k+1}{2}+\left(\mu+\frac{3 k-3}{2}\right)_{+, \varepsilon}}\left(S_{1}, S_{2}\right)+I^{\mu-2, \mu-1+(-1+k)_{+, \varepsilon}}\left(S_{1}, S_{2}\right) \\
& +I^{-k-2+(\mu+k)_{+, \varepsilon}, \mu-2+k}\left(S_{1}, S_{2}\right)+H_{\text {loc }}^{s_{0}+1-(\mu+k)_{+}},
\end{aligned}
$$

with the wavefront set of the last term contained in $L$. Given the $\Lambda_{\square}$-invariant neighborhood $L$ and any integer $N \geqq 1$, we can find another $\Lambda_{\square}$ invariant neighborhood, $L_{N} \Subset L$ such that $W F\left(\left(\square^{-1} M_{q}\right)^{N}\right)\left(L_{N}\right) \subset L$. Making all of the above microlocalizations away from $L_{N}$, and applying $\sum_{j=0}^{N-1}(-1)^{j}\left(\square^{-1} M_{q}\right)^{j}$ to both sides of (3.48), one sees using Lemmas 1.2, 1.3, 1.4 and 1.5 that, since $\mu<1-k$, the right side of (3.48) is stable under the application of $\left(\square^{-1} M_{q}\right)^{j}$ for $0 \leqq j \leqq N-1$. Thus, $\left(I+(-1)^{N}\left(\square^{-1} M_{q}\right)^{N}\right) \bar{u}$ belongs to the space on the right side of (3.48), and the wavefront set of the Sobolev space term is contained in $L$. Now apply $\rho^{*} R$ to both sides; using the mapping properties of $R$ and $\rho^{*}$, noting that $C_{\rho} \circ \Lambda_{1}=\emptyset$ for $t \gg 0$ (from (3.36)), and applying the standard Sobolev restriction theorem for hypersurfaces, we find that

$$
\rho^{*} F \bar{u} \in I^{-\frac{7}{4}}\left(\hat{\Lambda}_{+}\right)+I^{\mu+\frac{k}{2}-\frac{9}{4}}\left(\hat{\Lambda}_{-}\right)+H_{\mathrm{loc}}^{r_{0}+\delta N+n-\frac{3}{2}}
$$

where $\bar{u} \in H_{\text {loc }}^{r_{0}}$ and $\delta=1-(\mu+k)_{+}>0$.

Taking $N \rightarrow+\infty$, we thus have shown

Theorem 3.1. Microlocally away from $\hat{L}$,

$$
\alpha(s, \theta, \omega)-\rho^{*} F\left(u_{0}+u_{1}\right) \in I^{-\frac{7}{4}}\left(\hat{\Lambda}_{+}\right)_{+}+I^{\mu+\frac{k}{2}-\frac{9}{4}}\left(\hat{\Lambda}_{-}\right) .
$$

Thus, $\alpha \in I^{\frac{1}{4}}\left(\hat{\Lambda}_{+} \backslash \hat{L}\right)+I^{\mu+\frac{k}{2}-\frac{5}{4}}\left(\hat{\Lambda}_{-} \backslash \hat{L}\right)$, and hence $\hat{\Lambda}_{-}$and the principal symbol $\left.\sigma\left(\mu_{1}\right)\right|_{\hat{\Lambda}_{-}}$are determined by $\alpha$. 


\section{Determination of $S$ and $\sigma(q)$}

We now examine how the leading singularity of $q(x)$ is determined by the singularities of $\alpha(s, \theta, \omega)$ and its restriction to various submanifolds of $\mathbb{R} \times S^{n-1} \times S^{n-1}$. We start by showing that $\alpha(s, \theta, \omega)$ determines the surface, $S$. By Theorem 3.1, it suffices to show that $S$ is determined by $\hat{\Lambda}_{-}$, where $\hat{\Lambda}_{-}$is given by $(3.43)_{1}$ and (3.43) $)_{k}$ in the cases where the codimension of $S$ is 1 and greater than 1 , respectively. For simplicity, consider $k=1$ first; suppose there are two hypersurfaces, $S, \bar{S}$, such that $\hat{\Lambda}_{-}=\hat{\Lambda}_{-}$. Then, by $(3.43)_{1}$, there is a mapping $\bar{y}: S \times S^{n-1} \rightarrow \bar{S}$, such that

$$
\begin{aligned}
& \left\{\left(-\bar{y} \cdot(\bar{v}+\omega),-\bar{v}, \omega ; \sigma,-\sigma i_{-\bar{v}}^{*} \bar{y}, \sigma i_{\omega}^{*} \bar{y}\right)\right\} \\
& \quad=\left\{\left(-x \cdot(v+\omega),-v, \omega ; \sigma,-\sigma i_{-}^{*} y, \sigma i_{\omega}^{*} y\right)\right\},
\end{aligned}
$$

where $\bar{v}=\bar{v}(\bar{y}(, \omega), \omega)$. Identifying the second $(\theta)$ coordinates in (4.1) yields $2\left(\bar{h}_{\bar{y}} \cdot \omega\right) \bar{h}_{\bar{y}}-\omega=2\left(h_{y} \cdot \omega\right) h_{y}-\omega \Rightarrow\left(\bar{h}_{\bar{y}} \cdot \omega\right) \bar{h}_{\bar{y}}=\left(h_{y} \cdot \omega\right) h_{y}$; since $h_{y}$ and $\bar{h}_{\bar{y}}$ are unit vectors, this $\Rightarrow$

$$
\overline{h_{\bar{y}}}= \pm h_{y} .
$$

Identifying the last $(\Omega)$ components in (4.1) yields $i_{\omega}^{*} \bar{y}=i_{\omega}^{*} y \Rightarrow$

$$
\bar{y}=y+c_{1} \omega, \quad c_{1}=c_{1}(y, \omega) .
$$

Finally, comparing the first (s) components, we have

$$
\bar{y} \cdot(\bar{v}+\omega)=y \cdot(v+\omega) \Rightarrow \bar{y} \cdot\left(\overline{h_{\bar{y}}} \cdot \omega\right) \overline{h_{y}}=y \cdot\left(h_{y} \cdot \omega\right) h_{y} \Rightarrow
$$

by (4.2), $\bar{y} \cdot\left( \pm h_{y} \cdot \omega\right)\left( \pm h_{y}\right)=y \cdot\left(h_{y} \cdot \omega\right) h_{y} \Rightarrow$ by $(4.3),\left(y+c_{1} \omega\right) \cdot\left(h_{y} \omega\right) h_{y}=y\left(h_{y} \cdot \omega\right) h_{y}$. Now, $h_{y} \cdot \omega$ is not identically 0 on $S \times S^{n-1}$, so this $\Rightarrow\left(y+c_{1} \omega\right) \cdot h_{y}=y \cdot h_{y}$ $\Rightarrow c_{1} \omega \cdot h_{y}=0$. But again, $\omega \cdot h_{y} \not \equiv 0$, so this $\Rightarrow c_{1}=0 \Rightarrow \bar{y}=y \Rightarrow \bar{S}=S$.

For $1<k \leqq n$, we repeat the above argument, substituting $(3.43)_{k}$ for $(3.43)_{1}$. Thus, there is a function $(\bar{y}, \bar{v}):\left(N^{*} S \backslash 0\right) \times S^{n-1} \rightarrow N^{*} \bar{S} \backslash 0$ making the identification between $\hat{\Lambda}_{-}$and $\overline{\hat{\Lambda}}_{-}$, and we may assume $\bar{v}(y, \omega)^{2}=v^{2}$. Identifying the $\theta$-coordinates then yields $2(v \cdot \omega) \frac{v}{v^{2}}=2(\bar{v} \cdot \omega) \frac{\bar{v}}{\bar{v}^{2}}$, which implies $v= \pm \bar{v}, \tau= \pm \bar{\tau}$. The rest of the proof is the same.

We next consider the restriction of $\alpha(s, \theta, \omega)$ to submanifolds. Consider first the case of backscattering.

Let $\mathbb{B}=\{(s, \theta, \omega): \theta=-\omega\} \subset \mathbb{R} \times S^{n-1} \times S^{n-1}$ be the backscattering surface. Let $j_{\mathbb{B}}: \mathbb{R} \times S^{n-1} \rightarrow \mathbb{B}$ be the canonical parametrization, $j_{B}(s, \omega)=(s,-\omega, \omega)$; then the pullback operator,

$$
j_{\mathbb{B}}^{*}: \mathscr{D}^{\prime}\left(\mathbb{R} \times S^{n-1} \times S^{n-1}\right) \rightarrow \mathscr{D}_{B}^{\prime}\left(\mathbb{R} \times S^{n-1}\right),
$$

defined on distributions whose wavefronts sets are disjoint from the normals of $j_{B}$, is a Fourier integral operator, $j_{\mathbb{B}}^{*} \in I^{\frac{n-1}{4}}\left(C_{\mathbb{B}}\right)$, where

$$
\begin{gathered}
C_{\mathbb{B}}=\left\{\left(s, \omega, \sigma, \Omega ; s^{\prime}, \theta, \omega^{\prime}, \sigma^{\prime}, \Theta, \Omega^{\prime}\right): s=s^{\prime}, \omega=\omega^{\prime}=-\theta,\right. \\
\left.(\sigma, \Omega)=\left(\sigma^{\prime}, \Theta, \Omega^{\prime}\right) / N_{(s,-\omega, \omega)}^{*} \mathbb{B}\right\} .
\end{gathered}
$$

Let $L_{\mathbb{B}}=C_{\mathbb{B}} \circ \hat{L} \subset T^{*} \mathbb{B} \backslash 0$. 
Theorem 4.1. Away from $L_{\mathbb{B}}$,

$$
\left.\alpha\right|_{\mathbb{B}} \in I^{\mu+\frac{k-3}{2}+\frac{n}{4}}\left(\Lambda_{\mathbb{B}}\right)
$$

where

$$
\begin{aligned}
\Lambda_{\mathbb{B}}= & \left\{\left(-2 y \cdot h_{y}, h_{y} ; \sigma, \sigma i_{h_{y}}^{*} y\right): y \in S, \sigma \in \mathbb{R} \backslash 0\right\} \\
& \cup\left\{\left(2 y \cdot h_{y},-h_{y} ; \sigma, \sigma i_{h_{y}}^{*} y\right): y \in S, \sigma \in \mathbb{R} \backslash 0\right\} \quad(k=1)
\end{aligned}
$$

and

$$
\Lambda_{\mathbb{B}}=\left\{\left(-2 y \cdot \frac{v}{|v|}, \frac{v}{|v|} ;|v|,|v| \underset{\frac{v}{|v|}}{i_{v}^{*}} y\right):(y, v) \in N^{*} S \backslash 0\right\}, \quad 1<k \leqq n-1 .
$$

Proof. $\left.\alpha\right|_{\mathbb{B}}=j_{\mathbb{B}}^{*}(\alpha) \in I^{\mu+\frac{k}{2}-\frac{5}{4}+\frac{n-1}{4}}\left(C_{\mathbb{B}} \circ \hat{\Lambda}_{-}\right)$by the transverse intersection calculus, provided a) $\left.\hat{\Lambda}_{-} \pitchfork T^{*}\left(\mathbb{R} \times S^{n-1} \times S^{n-1}\right)\right|_{\mathbb{B}}$ and b) $\hat{\Lambda}_{-} \cap N^{*} \mathbb{B}=\emptyset$; both of these, together with $(4.6)_{1},(4.6)_{k}$ follow from $(3.43)_{1},(3.43)_{k}$, respectively. Q.E.D.

Corollary 4.2. $\left.\alpha\right|_{\mathrm{B}}$ determines $S$.

Proof. We deal with the case $k=1$, the case $1<k \leqq n$ being handled similarly. By Theorem $4.1,\left.\alpha\right|_{\mathbb{B}}$ is, microlocally away from $L_{\mathbb{B}}$, a Fourier integral distribution associated with a lagrangian $\Lambda_{B} \subset T^{*} \mathbb{B} \backslash 0$, invariant under the canonical involution induced by $(s, \omega) \rightarrow(-s, \omega)$. On either component, we see that

$$
\frac{\Omega}{\sigma}-\frac{1}{2} s \omega=i_{ \pm h_{y}}^{*} y \pm\left(x \cdot h_{y}\right) h_{y}=y .
$$

Thus, $S=\left\{\frac{\Omega}{\sigma}-\frac{1}{2} s \omega:(s, \omega, \sigma, \Omega) \in \Lambda_{B}\right\}$. Q.E.D.

Now consider a more general manifold of scattering data, $\mathbb{D}=$ $\{(s, \theta, \omega): \theta=\phi(\omega)\}$, where $\phi=S^{n-1} \rightarrow S^{n-1}$ is smooth. By (3.43) 1 , at a point of $\tilde{\Lambda}_{-} \cap T^{*}\left(\mathbb{R} \times S^{n-1} \times S^{n-1}\right)$, we must have

$$
\omega-2\left(\omega \cdot h_{y}\right) h_{y}=\phi(\omega)
$$

or

$$
\frac{1}{2}\|\omega-\phi(\omega)\|\left(\frac{\omega-\phi(\omega)}{\|\omega-\phi(\omega)\|}\right)=\left(\omega \cdot h_{y}\right) h_{y} .
$$

Letting

$$
\varphi(\omega)=\frac{\omega-\phi(\omega)}{\|\omega-\phi(\omega)\|}
$$

under the assumption $\phi(\omega) \neq \omega, \forall \omega \in S^{n-1}$, we thus have

$$
\varphi(\omega)= \pm h_{y} .
$$

We now assume that $\varphi$ has a smooth left inverse, at least on the image of the Gaussian map of $S$. Then the image of $\hat{\Lambda}_{-}$under intersection with $\left.T^{*}\left(\mathbb{R} \times S^{n-1} \times S^{n-1}\right)\right|_{\mathbb{D}}$ and modding out by $N^{*} \mathbb{D}$ is (in $(s, \omega, \sigma, \Omega)$ coordinates),

$$
\Lambda_{\mathbb{D}}=\left\{\left(-2\left(\varphi^{-1}\left( \pm h_{y}\right) \cdot h_{y}\right) y \cdot h_{y}\right), \varphi^{-1}\left( \pm h_{y}\right) ; \sigma, \sigma i_{\varphi^{-1}}^{*}\left( \pm h_{y}\right): y \in S, \sigma \in \mathbb{R} \backslash 0\right\} .
$$


As in the proof of Corollary 4.2, we can reconstruct $y \in S$ from $\frac{\Omega}{\sigma}=i_{\varphi^{-1}\left( \pm h_{y}\right)}^{*} y$, the projection of $y$ onto $\varphi^{-1}\left( \pm h_{y}\right)^{\perp}$, and the dot product of $y$ with any vector not in $\varphi^{-1}\left( \pm h_{y}\right)^{\perp}$; but, if $\varphi^{-1}(v) \cdot v \neq 0$ for all $v$ in the image of the Gaussian map of $S$, then this is determined by the $S$ component.

We thus have established, for $k=1$,

Corollary 4.3. If $\mathbb{D}=\{(s, \theta, \omega): \theta=\phi(\omega)\}$ with

a) $\phi(\omega) \neq \omega, \forall \omega \in S^{n-1}$,

b) $\varphi(\omega)=\frac{\omega-\phi(\omega)}{\|\omega=\phi(\omega)\|}$ a diffeomorphism,

c) $\varphi^{-1}(v) \cdot v \neq 0, v \in$ Gaussian image of $S$, then $\left.\alpha\right|_{\mathbb{D}}$ determines $S$.

Finally, we note that Corollary 4.3 holds for $D$ of the form $\mathbb{D}=\{(s, \theta, \omega): \theta=\phi(s, \omega)\}$, satisfying

$\left.\mathrm{a}^{\prime}\right) \varphi(s, \omega) \neq \omega, \forall \omega \in S^{n-1}$.

$\left.\mathrm{b}^{\prime}\right) \varphi(s, \cdot)=\frac{\cdot-\phi(s, \cdot)}{\|\cdot-\phi(s, \cdot)\|}$ is a diffeomorphism $S^{n-1} \rightarrow S^{n-1}$ for all $s \in \mathbb{R}$.

c $) ~^{-1}(s, v) \cdot v \neq 0, \forall v \in$ Gaussian image, $s \in \mathbb{R}$.

We leave the statement of Corollary 4.3 for $1<k \leqq n$ to the interested reader.

Finally, we show that for determined sets $\mathbb{D}$ of scattering data as above, the principal symbol $\left.\sigma(q)\right|_{N^{*} S}$ is determined by the principal symbol $\left.\sigma\left(\left.\alpha\right|_{\mathbb{D}}\right)\right|_{\Lambda_{\mathbb{D}}}$. For simplicity, we work with $\mathbb{D}=\mathbb{B}$, the backscattering. In fact, by Theorem 3.1 , the principal symbol of $\alpha$ on $\hat{\Lambda}_{-} \backslash \hat{L}$ is the same as that of $\rho^{*} F\left(u_{1}\right)=-\rho^{*} F \square^{-1}(q \cdot \delta)$. Microlocally near $\Sigma_{-}$, the principal symbol of $q(x) \cdot \delta(t-x \cdot \omega)\left(\right.$ in $\left.I^{\mu+1-\frac{n}{\Sigma}}\left(\Lambda_{2}\right)\right)$ is

$$
\sigma(q)(x, v) \cdot 1(\tau)
$$

in the coordinates of (3.13). By Proposition 2.1, the symbol of $\square^{-1}(q \cdot \delta)$ at a point $(x, \tau, \omega ; \xi, \tau, \Omega) \in \hat{\Lambda}_{-} \backslash \hat{\hat{L}}$ is proportional $\left(\right.$ by $\left.\sigma\left(\square^{-1}\right)\right)$ to the symbol of $q \cdot \delta$ at that point of $\Sigma_{-}$on the same bicharacteristics. Since the Fourier integral operators $F, \rho^{*}$ and $j_{\mathbb{B}}^{*}$ are elliptic, the symbol

$$
\sigma\left(\left.\alpha\right|_{\mathbb{B}}\right)\left( \pm 2 y \cdot h_{y}, \pm h_{y} ; \sigma, \sigma i_{ \pm}^{*} h_{y} y\right) \quad(k=1)
$$

or

$$
\sigma\left(\left.\alpha\right|_{\mathbb{B}}\right)\left(-2 y \cdot \frac{v}{|v|}, \frac{v}{|v|} ;|v|,|v|_{\frac{v}{|v|}}^{*} y\right) \quad(1<k \leqq n)
$$

is proportional to $\sigma(q)(y, v)$; since $\Sigma_{-} \backslash \Sigma_{2}$ is dense in $\Sigma_{-}$, we can recover $\sigma(q)$ on all of $N * S \backslash 0$.

\section{References}

[BLM] Bayliss, A., Lin, Y., Morawetz, C.: Scattering by a potential using hyperbolic methods. Math. Comp. 52, 321-338 (1989)

[BC] Beals, R., Coifman, R.: Multidimensional inverse scattering and nonlinear PDE. Proc. Symp. Pure Math. 43, 45-70 (1985) 
[DG] Duistermaat, J.J., Guillemin, V.: The spectrum of positive elliptic operators and periodic bicharacteristics. Invent. Math. 29, 39-79 (1975)

[DH] Duistermaat, J.J., Hörmander, L.: Fourier integral operators, II. Acta. Math. 128, 183-269 (1972)

[ER] Eskin, G., Ralston, J.: The inverse backscattering problem in three dimensions. Commun. Math. Phys. 124, 169-215 (1989)

[GU] Greenleaf, A., Uhlmann, G.: Estimates for singular Radon transforms and pseudodifferential operators with singular symbols. J. Funct. Anal. 89, 202-232 (1990)

[GuU] Guillemin, V., Uhlmann, G.: Oscillatory integrals with singular symbols. Duke Math. J. 48, 251-267 (1981)

[HN] Henkin, G., Novikov, R.: A multidimensional inverse problem in quantum and acoustic scattering. Inverse Prob. 4, 103-121 (1988)

[Hö] Hörmander, L.: Fourier integral operators, I. Acta Math. 127, 79-183 (1971)

[LP] Lax, P., Phillips, R.: Scattering Theory. Revised Edition. New York, London: Academic Press 1989

[MU1] Melrose, R., Uhlmann, G.: Lagrangian intersection and the Cauchy problem. Comm. Pure Appl. Math. 32, 482-512

[MU2] Melrose, R., Uhlmann, G.: Introduction to Microlocal Analysis with Applications to Scattering Theory. Book in preparation

[N] Nachman, A.: Inverse Scattering at fixed energy. Proc. Int. Cong. Math. Phys. Leipzig, 1991 (to appear)

[No] Novikov, R.: Multidimensional inverse spectral problem for the equation $-\Delta \psi+(V(x)-E u(x)) \psi=0$. Funct. Anal. Appl. 22, 263-272 (1988)

[Pe] Petkov, V.: Scattering theory for hyperbolic operators. Amsterdam: North-Holland 1989

[P] Phillips, R.: Scattering theory for the wave equation with a short range potential. Indiana Univ. Math. J. 31, 609-639 (1982)

[PäS] Päivärinta, L., Somersalo, E.: Inversion of discontinuities for the Schrödinger operator in three dimensions. SIAM J. Math. Anal. 22, 480-499 (1991)

[Pi] Piriou, A.: Calcul symbolique non lineare pour une onde conormale simple. Ann. Inst. Four. 38, 173-188 (1988)

[Pr] Prosser, R.J.: Formal solutions of inverse scattering problems, III and IV. J. Math. Phys. 21, 2648-2653 (1980) and 23, 2127-2130 (1982)

Communicated by B. Simon 